\title{
ON THE FUNCTIONS OF LITTLEWOOD-PALEY, LUSIN, AND MARCINKIEWICZ
}

\author{
BY \\ E. M. STEIN
}

1. Introduction. In their work on Fourier series Littlewood and Paley [5], introduced the function $g$ as follows:

$$
g(f)(\theta)=\left(\int_{0}^{1}(1-\rho)\left|\Phi^{\prime}\left(\rho e^{i \theta}\right)\right|^{2} d \rho\right)^{1 / 2} .
$$

$\Phi(z)$ is the function which is analytic in $|z|<1$, and whose real part has boundary value $f(\theta)$. One of their main results is $\left({ }^{1}\right)$ :

$$
\|g(f)\|_{p} \leqq A_{p}\|f\|_{p}, \quad 1<p<\infty .
$$

Accompanying this, they also proved the following "converse" result:

$$
\|f\|_{p} \leqq A_{p}\|g(f)\|_{p}
$$

$1<p<\infty$

where it is assumed that

$$
\int_{0}^{2 \pi} f(\theta) d \theta=0
$$

In an earlier study on boundary values of analytic functions, Lusin [6] introduced the function

$$
S(\Phi)(\theta)=\left(\iint_{\Omega(\theta)}\left|\Phi^{\prime}\right|^{2} d \omega\right)^{1 / 2} .
$$

Here, $\Omega(\theta)$ is a standard "triangular" domain inside the unit circle whose vertex is at the point $\theta ; d \omega$ is the Euclidean element of area $\left({ }^{2}\right)$.

Marcinkiewicz and Zygmund [9], proved that

$$
\|S(\Phi)\|_{p} \leqq A_{p}\|\Phi\|_{p}, \quad 0<p<\infty .
$$

From this it follows, by a well-known theorem of M. Riesz, that:

$$
\|S(\Phi)\|_{p} \leqq A_{p}\|f\|_{p}, \quad 1<p<\infty .
$$

Received by the editors December 22, 1956.

(1) Here, as elsewhere, $A_{p}$ stands for a general constant, depending on the indicated parameter. The constant $A_{p}$ need not be the same at different occasions.

(2) Marcinkiewicz and Zygmund [9], noted that $S$ is essentially a majorant of $g$. We refer the reader to [14 Chapter XIV], for further facts concerning the function $S(\Phi)$. 
Finally, in this connection, Marcinkiewicz [7] introduced the function $\mu(f)$ :

$$
\mu(f)=\left(\int_{0}^{2 \pi} \frac{|F(x+t)+F(x-t)-2 F(x)|^{2}}{t^{3}} d t\right)^{1 / 2}
$$

where $F(x)=\int_{0}^{x} f(t) d t$.

The function $\mu(f)$ was introduced in order to give an analogue of the function $g(f)$, without however going into the interior of the unit circle for its definition.

Marcinkiewicz conjectured, and Zygmund proved [11], that:

$$
\|\mu(f)\|_{p} \leqq A_{p}\|f\|_{p}
$$$$
1<p<\infty
$$

and

$$
\|f\|_{p} \leqq A_{p}\|\mu(f)\|_{p}
$$$$
1<p<\infty
$$

if, $\int_{0}^{2 \pi} f(\theta) d \theta=0$.

The literature in question reveals that these results are closely interdependent. Moreover, all proofs given depend, at crucial points, on "complexvariable" methods.

It is our intention here to attack these problems by the methods of realvariable theory, and to extend these results to higher dimensions.

It turns out that the real-variable techniques used in the study of the "Hilbert transform"

$$
\tilde{f}(x)=\mathrm{P} . \mathrm{V} \cdot \int_{0}^{\infty} \frac{f(x+t)-f(x-t)}{t} d t
$$

are adaptable for our purposes. The motivation for this lies in the fact (1.4) may be rewritten as:

$$
\tilde{f}(x)=\int_{0}^{\infty} \frac{(F(x+t)+F(x-t)-2 F(x))}{t^{2}} d t .
$$

It is to be noted that $\left(1.4^{*}\right)$ resembles $(1.3)$ in form.

In dealing with generalizations of the functions $g, S$, and $\mu$ to higher dimensions, we are guided by the techniques used by A. P. Calderón and A. Zygmund $\left(^{3}\right)$ in their study of the $n$-dimensional generalizations of the Hilbert transform (1.4); connected with this are some earlier ideas of Marcinkiewicz $\left({ }^{4}\right)$. We should mention at this point that a generalization of the function $S$ similar to the one we shall consider had been previously studied by A. P. Calderón [1] in a different setting.

Another remark concerning the methodology of this paper is in order. The

(3) See especially [2] and [3].

(4) See [7] and [8]. 
deeper real-variable discussion is contained principally in Lemmas 7 and 12, with the aid of Lemmas 1, 2, and 3. These lemmas allow us to prove inequalities analogous to $(A)$, and $(C)$, for $1<p \leqq 2$. In the classical situation this is exactly the point where the complex method was indispensable, especially in the use of the "Blaschke product" decomposition of analytic functions. Thus, at this point is contained the main contributions of this paper. The rest of the inequalities are then deduced-in the classical situation and as they are here-by methods more properly from the domain of harmonic functions than analytic functions. Here, our only contribution is to point out how the classical proofs may be extended to $n$-dimensions. Whatever difficulties involved here are surmounted by using the theory of singular integrals as the appropriate substitute for the notion of the conjugate function.

The plan of this paper is as follows: $\$ 2$ will be devoted to a review of the basic facts and techniques needed, all of which were previously known. $\$ \S 3$ and 4 will contain the generalizations of the function $\mu$ of Marcinkiewicz, and the proof of an inequality analogous to $(C) . \S \S 5$ and 6 will be devoted to the extensions of the functions $g$ and $S$ to higher dimensions, and the proofs of inequalities like (A) and (B). In $\S 7$ we turn to the "converse" inequalities, and establish an appropriate analogue to $\left(A^{*}\right)$, and in $\S 8$, we discuss analogues to $\left(\mathrm{C}^{*}\right)$.

2. Background facts. In what follows, we shall use the following $n$-dimensional notation. $x, y, z, \cdots$ will denote points (vectors) in Euclidean $n$ space, $E_{n}$. In coordinate notation we write $x=\left(x_{1}, x_{2}, \cdots, x_{n}\right) ; d x$ denotes the element of Euclidean $n$-dimensional volume; $|x|$ denotes the length of the vector $x$, i.e. $|x|^{2}=x_{1}^{2}+x_{2}^{2} \cdots+x_{n}^{2} ; x^{\prime}$ denotes the unit vector in the direction of $x$, i.e. $x^{\prime}=x /|x| ; \Sigma$ is the unit sphere, $|x|=1$; and $d \Sigma$ is the Euclidean element of measure on $\Sigma$.

For any measurable $E$, let $|E|$ denote its Euclidean-Lebesgue measure. $L_{p}\left(E_{n}\right)$ will denote the class of functions whose $p$ th power is integrable with respect to Euclidean measure, and for any such function $f(x),\|f\|_{p}$ will denote $\left(\int_{E_{n}}|f(x)|^{p} d x\right)^{1 / p}$.

The following "covering" lemma is basic to the real-variable technique that follows. It is a generalization of a well-known lemma of F. Riesz in one dimension; the $n$-dimensional form that follows is due to A. P. Calderón and A. Zygmund. We have restated their lemma differently, combining statements found in the proof of several of their lemmas. (The relevant material is found in [2, pp. 91-94].)

LEMMA 1. Let $f(x) \geqq 0$, and suppose $\int_{E_{n}} f(x) d x<\infty$.

Let there be given an $s>0$. Then there exists an open set $E_{s}$, and a decomposition of $f(x), f(x)=g(x)+h(x)$, with the following properties:

(1) $\left|E_{s}\right| \leqq(1 / s) \int_{E_{n}} f(x) d x$.

(2) $E_{s}$ is the union of disjoint open cubes $I_{k}, k=1,2, \ldots$. 
(3) $h(x)=f(x)$, for $x \notin E_{s}$, and for those $x, 0 \leqq h(x) \leqq s$.

(4) $|h(x)| \leqq 2^{n} \cdot s$, for $x \in E_{s}$.

(5) $g(x)=0$, for $x \in E_{s}$.

(6) $\int_{I_{k}} g(x) d x=0$, for each of the open cubes $I_{k}$ whose union is $E_{s}$.

Proof. The proof is an immediate combination of Lemma 1 of [2], and the decomposition that follows, made at the top of p. 94 .

The following lemma is related to the previous. It is a generalization of the Hardy-Littlewood "maximal theorem" and F. Riesz's lemma.

Lemma 2. Let $f(x) \in L_{p}\left(E_{n}\right), 1 \leqq p \leqq \infty$. Define $f^{*}(x)$ to be

$$
\sup _{t>0} \frac{1}{t^{n}} \int_{|y| \leqq t}|f(x+y)| d y .
$$

Then if $f(x) \in L_{p}\left(E_{n}\right), 1<p$, so is $f^{*}(x)$, and

$$
\left\|f^{*}(x)\right\|_{p} \leqq A_{p}\|f(x)\|_{p},
$$

Moreover, if $f(x) \in L_{1}\left(E_{n}\right)$, then $f^{*}(x)$ is finite almost everywhere; and if $s>0$, then $\left({ }^{5}\right)$ :

$$
\left|\left\{f^{*}(x)>s\right\}\right| \leqq(A / s) \int_{E_{n}}|f| d x .
$$

For a proof see [2] pp. 114-116.

The functions $g, S$, and $\mu$, may be thought of as operators-although as nonlinear ones. An "interpolation" theorem of Marcinkiewicz is very useful in this connection.

In quoting the result of Marcinkiewicz-which has been extended by A. Zygmund $\left({ }^{6}\right)$-we shall not aim at generality. For the sake of simplicity we shall limit ourselves to the special case that is needed.

Let $T$ be an operator whose domain and range are functions on $E_{n}$. Suppose that $T$ satisfies the following two conditions:

$$
\begin{aligned}
& T(f) \text { is defined for every } f \in L_{p}\left(E_{n}\right), 1 \leqq p . \\
& \left|T\left(f_{1}+f_{2}\right)\right| \leqq\left|T\left(f_{1}\right)\right|+\left|T\left(f_{2}\right)\right| .
\end{aligned}
$$

For a given $p, 1 \leqq p<\infty$, we shall say that an operator satisfying the above conditions is of weak type $p$, if

$$
|\{|T f|>s\}| \leqq\left(A / s^{p}\right) \int_{E_{n}}|f|^{p} d x
$$

for all $s, 0<s<\infty$, all $f \in L_{p}\left(E_{n}\right)$ and some $A$ independent of $f$ and $s$.

(5) The notation $|\{\cdots\}|$ indicates: "the measure of the set."

(6) See [13], where a proof of the general interpolation theorem is found. 
We shall say that the operator $T$ is of strong type $p$, if for some fixed $p$, $1 \leqq p:$

$$
\int_{E_{n}}|T f|^{p} d x \leqq A \int_{E_{n}}|f|^{p} d x, \quad \quad f \in L_{p}\left(E_{n}\right)
$$

where $A$ is independent $\left({ }^{7}\right)$ of $f$.

We are now in a position to state the result of Marcinkiewicz:

Lemma 3. Let $T$ be an operator which satisfies conditions (2.1) and (2.2). Given two indices $p_{1}$, and $p_{2}, 1 \leqq p_{1}<p_{2}<\infty$, suppose that $T$ is simultaneously of weak type $p_{1}$, and weak type $p_{2}$.

ConcLusion: $T$ is of strong type $p$ for each $p, p_{1}<p<p_{2}$.

The following lemma-due to Calderón and Zygmund-is one of the principal results in their theory of "singular integrals" which generalize the one-dimensional "Hilbert transform." $\left.{ }^{8}\right)$

Let $\Omega(x)$ be a function for $x \in E_{n}$, which is homogeneous of degree 0 , i.e. depends only on the direction of the vector. Suppose that $\Omega$ satisfies the following two conditions:

(2.5) $\Omega$ is continuous on $\Sigma$, and satisfies there a Lipschitz condition of order $\alpha, 0<\alpha \leqq 1$.

$$
\int_{\Sigma} \Omega\left(x^{\prime}\right) d \Sigma=0
$$

Lemma 4. Let $\Omega$ satisfy the conditions (2.5) and (2.6) above. If $\tilde{f}_{\epsilon}(x)$ $=\int_{|y|>\epsilon}\left(\Omega\left(y^{\prime}\right) /|y|^{n}\right) f(x-y) d y$ and $f(x) \in L_{p}\left(E_{n}\right), 1 \leqq p<\infty$, then

$$
\lim _{\epsilon \rightarrow 0} \tilde{f}_{\epsilon}(x)=\tilde{f}(x) \text { exists almost everywhere, }
$$

and

$$
\|\tilde{f}(x)\|_{p} \leqq A_{p}\|f(x)\|_{p}
$$$$
1<p<\infty,
$$

where $A_{p}$ is independent of $f$.

3. The Marcinkiewicz integral; case when $1 \leqq p \leqq 2$. We shall now define the generalization of the Marcinkiewicz integral (1.3) whose properties we shall study. As stated previously, this generalization is analogous to the generalization of the Hilbert-transform given by Calderón and Zygmund (Lemma 4).

Let $\Omega(x)$ be a function which is homogeneous of degree 0 , and which

(7) It is easy to check that if $T$ is of strong type $p$, then it is also of weak type $p$, and the constant $A$ which appears in (2.3) may be taken to be the constant $A$ which appears in (2.4).

$\left.{ }^{8}\right)$ Again, we shall not state the results in their most general form. The result of Lemma 4 is proved in [2], and generalizations may be found in [3]. 
satisfies conditions (2.5) and (2.6). For any $f(x) \in L_{p}\left(E_{n}\right), 1 \leqq p$ define $F_{t}(x)$, by

$$
F_{t}(x)=\int_{|y| \leqq t} f(x-y) \frac{\Omega\left(y^{\prime}\right)}{|y|^{n-1}} d y .
$$

We note, in the case $n=1$, if $\Omega\left(y^{\prime}\right)=\operatorname{sign} y$, then $F_{t}(x)=F(x+t)+F(x-t)$ $-2 F(x)$, where $F(x)=\int_{0}^{x} f(s) d s$. Define now $\mu(f)$, by

$$
\mu(f)(x)=\left(\int_{0}^{\infty} \frac{\left|F_{t}(x)\right|^{2}}{t^{3}} d t\right)^{1 / 2} .
$$

We prove first:

Lemma 5. With $\Omega$ satisfying the above conditions, and $\mu(f)$ defined by (3.2),

$$
\int_{E_{n}}(\mu(f)(x))^{2} d x \leqq A \int_{E_{n}}|f(x)|^{2} d x,
$$

where $A$ is independent of $f$.

Proof. We note first, that by (3.1), $F_{t}(x)$ is for each $t$ the convolution of $f(x)$ with an integrable function. Thus $F_{t}(x)$ is well defined for any $f(x)$ $\in L_{p}\left(E_{n}\right)$. Now

$$
\begin{aligned}
\int(\mu(f)(x))^{2} d x & =\iint_{0}^{\infty} \frac{\left|F_{t}(x)\right|^{2}}{t^{3}} d t d x \\
& =\int_{0}^{\infty} \frac{d t}{t^{3}}\left(\int_{E_{n}}\left|F_{t}(x)\right|^{2} d x\right),
\end{aligned}
$$

by Fubini's theorem.

We shall evaluate $\int_{E_{n}}\left|F_{t}(x)\right|^{2} d x$ by Plancherel's theorem.

Let $\hat{f}(x)=\int_{E_{n}} e^{-i x} \cdot y f(y) d y$ be the Fourier transform of $f(x)\left({ }^{9}\right)$. Also let:

$$
k_{t}(x)=\int_{|y| \leqq t} e^{-i x \cdot y} \frac{\Omega\left(y^{\prime}\right)}{|y|^{n-1}} d y .
$$

Then by Plancherel's theorem:

$$
\int_{E_{n}}\left|F_{t}(x)\right|^{2}=(2 \pi)^{n} \int_{E_{n}}\left|k_{t}(x)\right|^{2}|\hat{f}(x)|^{2} d x .
$$

We shall now make the following estimates on $k_{t}(x)$ :

$$
\begin{aligned}
& \left|k_{t}(x)\right| \leqq A|x| t^{2}, \\
& \left|k_{t}(x)\right| \leqq A /|x|, \text { if } t \geqq 1 /|x|, \text { and } n \geqq 3
\end{aligned}
$$

(9) The notation $x \cdot y$ denotes the inner product of the vectors $x$ and $y$. 
and

$\left(3.3^{*}\right) \quad\left|k_{t}(x)\right| \leqq A\left(1 /|x|+\frac{\log (|x| t)}{|x|}\right)$, if $t \geqq \frac{1}{|x|}$ and $n=2^{(10)}$.

Now

$$
k_{t}(x)=\int_{|y| \S t} e^{-i x \cdot y} \frac{\Omega\left(y^{\prime}\right)}{|y|^{n-1}} d y=\int_{|y| \leqq t}\left(e^{-i x \cdot y}-1\right) \frac{\Omega\left(y^{\prime}\right)}{|y|^{n-1}} d y,
$$

since

$$
\int_{|y| \leqq t} \frac{\Omega\left(y^{\prime}\right)}{|y|^{n-1}} d y=0
$$

by condition (2.6) imposed on $\Omega$.

Therefore $\left|k_{t}(x)\right| \leqq \int_{|y| \leqq t}|x| \cdot|y| \cdot\left(A /|y|^{n-1}\right) d y \leqq A|x| \int_{|y| \leqq t} d y /|y|^{n-2}$ $\leqq A|x| t^{2}$. The condition (3.2) is established.

In order to establish (3.3), let us introduce the following notation: $r=|x|, R=|y|$, and $\cos \left(x^{\prime}, y^{\prime}\right)=$ cosine of angle made by vectors $x^{\prime}$ and $y^{\prime}$.

Then

$$
\begin{aligned}
k_{t}(x) & =\int_{|y| \leqq t} e^{-i x \cdot y} \frac{\Omega\left(y^{\prime}\right)}{|y|^{n-1}} d y \\
& =\int_{\Sigma} \int_{0}^{t} e^{i r R \cos \left(x^{\prime}, y^{\prime}\right)} \Omega\left(y^{\prime}\right) d R d \Sigma^{\prime} \\
& =\int_{\Sigma}\left(\frac{e^{-i r t \cos \left(x^{\prime}, y^{\prime}\right)}-1}{r \cos \left(x^{\prime}, y^{\prime}\right)}\right) \Omega\left(y^{\prime}\right) d \Sigma .
\end{aligned}
$$

Thus

$$
\begin{aligned}
\left|k_{t}(x)\right| & \leqq \frac{1}{r} \cdot 2 \cdot \sup _{y^{\prime}}\left|\Omega\left(y^{\prime}\right)\right| \int_{\Sigma} \frac{d \Sigma}{\left|\cos \left(x^{\prime}, y^{\prime}\right)\right|} \\
& \leqq A / r=A /|x|
\end{aligned}
$$

since for $n \geqq 3, \int_{\Sigma} d \Sigma /\left|\cos \left(x^{\prime}, y^{\prime}\right)\right| \leqq A$.

This proves (3.3). In order to prove $\left(3.3^{*}\right)$ let us call $\phi$ the angle the vector $y^{\prime}$ makes with the axis $y_{1}$, and $\theta$ the angle the vector $x^{\prime}$ makes with the axis $x_{1}$.

Then proceeding as before, we find that

$$
k_{t}(x)=\int_{0}^{2 \pi}\left(\frac{e^{-i r t \cos (\theta-\phi)}-1}{r \cos (\theta-\phi)}\right) \Omega(\phi) d \phi .
$$

(10) For $n=1, \Omega(x)=\operatorname{sign} x$, and then $k_{t}(x)=C \cdot(1-\cos x t / x)$ so that (3.2) and (3.3) hold in this case. 
Thus, after an elementary change of variable,

$$
\begin{aligned}
k_{t}(x)= & \int_{-\pi / 2}^{+\pi / 2}\left(\frac{e^{-i r t \sin \phi}-1}{r \sin \phi}\right) \Omega(\theta-\phi+\pi / 2) d \phi \\
& -\int_{-\pi / 2}^{+\pi / 2}\left(\frac{e^{i r t \sin \phi}-1}{r \sin \phi}\right) \Omega(\theta-\phi+3 \pi / 2) d \phi .
\end{aligned}
$$

Each of these two integrals may be evaluated in the same manner. Thus

$$
\begin{aligned}
\int_{-\pi / 2}^{+\pi / 2}\left(\frac{e^{-i r t \sin \phi}-1}{r \sin \phi}\right) \Omega(\theta-\phi+\pi / 2) d \phi \\
=\int_{-\pi / 2}^{+\pi / 2}\left(\frac{e^{-i r t \sin \phi}-1}{r \sin \phi}\right)[\Omega(\theta-\phi+\pi / 2)-\Omega(\theta+\pi / 2)] d \phi \\
\quad+\Omega(\theta+\pi / 2) \int_{-\pi / 2}^{+\pi / 2}\left(\frac{e^{-i r t \sin \phi}-1}{r \sin \phi}\right) d \phi \\
=I_{1}+I_{2} .
\end{aligned}
$$

Owing to the Lipschitz condition on $\Omega$,

$$
\left|I_{1}\right| \leqq \frac{1}{r} \int_{-\pi / 2}^{+\pi / 2} \frac{A|\phi|^{\alpha}}{|\sin \phi|} d \phi \leqq A / r, \quad 0<\alpha \leqq 1 .
$$

Owing to the fact that $\left({ }^{11}\right)$,

$$
\left|\int_{-\pi / 2}^{+\pi / 2}\left(\frac{e^{i s \sin \phi}-1}{\sin \phi}\right) d \phi\right| \leqq A(1+\log |s|) \text { if }|s| \geqq 1
$$

then $\left|I_{2}\right| \leqq A(1+\log r t) / r$, if $r t \geqq 1$.

Collecting these estimates we get $\left|k_{t}(x)\right| \leqq A(1+\log r t) / r$, if $r t \geqq 1$, which proves $\left(3.3^{*}\right)$.

Now by $(3.1)$

$$
\begin{gathered}
\int_{E_{n}}(\mu(f))^{2} d x=\int_{0}^{\infty} \int_{E_{n}}\left|F_{t}(x)\right|^{2} d x \frac{d t}{t^{3}}=(2 \pi)^{n} \int_{E_{n}} d x \int_{0}^{\infty} \frac{\left|k_{t}(x)\right|^{2}}{t^{3}}|\hat{f}(x)|^{2} d t \\
=\int_{E_{n}}|\hat{f}(x)|^{2} d x \int_{0}^{1 /|x|} \frac{d t}{t^{3}}\left|k_{t}(x)\right|^{2}+\int_{E_{n}}|\hat{f}(x)|^{2} d x \int_{1 /|x|}^{\infty} \frac{d t}{t^{3}}\left|k_{t}(x)\right|^{2} .
\end{gathered}
$$

By (3.2), $\int_{0}^{1 /|x|}\left(d t / t^{3}\right)\left|k_{t}(x)\right|^{2} \leqq A$; and by (3.3) or $\left(3.3^{*}\right)$

$$
\int_{1 /|x|}^{\infty} \frac{d t}{t^{3}}\left|k_{t}(x)\right|^{2} \leqq A .
$$

(11) The estimate for $\int_{-\pi / 2}^{+\pi / 2}\left(e^{-i s \sin \phi}-1 / \sin \phi\right) d \phi$ is most easily made by breaking up the interval into $|\phi| \leqq 1 /|s|$, and a complementary part, where $|\phi|>1 /|s|$. 
Thus $\int_{E_{n}}(\mu(f))^{2} d x \leqq 2 A \int_{E_{n}}|\hat{f}(x)|^{2} d x=A^{\prime} \int_{E_{n}}|f(x)|^{2} d x$, and Lemma 5 is proved.

The following lemma is very straightforward.

Lemma 6. If $F_{t}(x)=\int_{|y| \leqq t} f(x-y)\left(\Omega\left(y^{\prime}\right) /|y|^{n-1}\right) d y$, as above, and $f^{*}(x)$ $=\sup _{t>0}\left(1 / t^{n}\right) \int_{|y| \leq t}|f(x-y)| d y$, then

$$
\sup _{t>0}\left|\frac{F_{t}(x)}{t}\right| \leqq A f^{*}(x) .
$$

Proof.

$$
\begin{aligned}
& \left|\frac{F_{t}(x)}{t}\right| \leqq \frac{1}{t} \int_{|y| \leqq t}|f(x-y)| \frac{\left|\Omega\left(y^{\prime}\right)\right|}{|y|^{n-1}} d y \leqq(A / t) \int_{|y| \leqq t} \frac{|f(x-y)|}{|y|^{n-1}} d y \\
& =(A / \imath) \sum_{k=0}^{\infty} \int_{2^{-k-1} t<|y| \leqq 2^{-k} t} \frac{|f(x-y)|}{|y|^{n-1}} d y \\
& \leqq\left(A / t^{n}\right) \sum \cdot 2^{(k+1)(n-1)} \int_{|y| \leqq 2^{-k} t}|f(x-y)| d y \\
& \leqq\left(A / l^{n}\right) \sum_{k=0}^{\infty} \cdot 2^{(k+1)(n-1)} \cdot 2^{-k n} \cdot l^{+n} \cdot f^{*}(x) \\
& \leqq A f^{*}(x) \sum_{k=0}^{\infty} 2^{(k+1)(n-1)} \cdot 2^{-k n}=2 A f^{*}(x) \sum_{k=0}^{\infty} 2^{-k} \\
& \leqq A^{\prime} f^{*}(x) \text {, }
\end{aligned}
$$

which proves the lemma.

Lemma 7. Let $f(x) \in L_{1}\left(E_{n}\right)$. Define $\mu(f)$ as in (3.2) above. Then $\mu(f)$ is finite almost everywhere. Moreover, if $s>0$, and $D_{s}$ is the set where $\mu(f)(x)>s$, then:

$$
\left|D_{s}\right| \leqq(A / s) \int_{E_{n}}|f(x)| d x .
$$

Proof $\left({ }^{12}\right)$. Without loss of generality, we shall restrict ourselves to a positive $f(x)$.

Given $s>0$, let $f(x)=g(x)+h(x)$ be the decomposition guaranteed by Lemma 1 , and $E_{s}$ the open set given by the lemma, which contains the support of $g(x)$. According to Lemma $1, h(x)=f(x)$ for $x \notin E_{s}$, and there $f(x) \leqq s$. Moreover, $|h(x)| \leqq 2^{n} s$ on $E_{s}$, and $\left|E_{s}\right| \leqq(1 / s) \int_{E_{n}} f(x) d x$.

\section{Thus}

(12) In this proof, we shall use the constants $A, c, \beta, \cdots$ repeatedly; as before, these constants may be different, at different occasions; their dependence on $n$ (the dimension of the space) will not be exhibited. 


$$
\begin{aligned}
\int_{E_{n}}|h(x)|^{2} d x & =\int_{E_{n}-E_{s}}|h(x)|^{2} d x+\int_{E_{s}}|h(x)|^{2} d x \\
& \leqq s \int_{E_{n}-E_{s}} h(x) d x+2^{2 n} s^{2}\left|E_{s}\right| \leqq\left(1+2^{2 n}\right) s \int_{E_{n}} f(x) d x .
\end{aligned}
$$

Thus $h(x) \in L_{2}\left(E_{n}\right)$, and

$$
\int_{B_{n}}|h(x)|^{2} d x \leqq c \cdot s \int_{E_{n}} f(x) d x .
$$

Similarly, $\int_{E_{n}}|g(x)| d x=\int_{E_{d}}|g(x)| d x \leqq \int_{E_{s}} f(x) d x+\int_{E_{a}}|h(x)| d x \leqq \int_{E_{n}} f(x) d x$ $+2^{n} s\left|E_{s}\right| \leqq\left(1+2^{n}\right) \int_{E_{n}} f(x) d x$. Thus $g(x) \in L_{1}\left(E_{n}\right)$, and:

$$
\int_{E_{n}}|g(x)| d x \leqq c \int_{E_{n}} f(x) d x .
$$

Now let $G_{t}(x)=\int_{|y| \leq t} g(x-y)\left(\Omega\left(y^{\prime}\right) /|y|^{n-1}\right) d y$, and

$$
H_{t}(x)=\int_{|y| \leqq t} h(x-y) \cdot\left(\Omega\left(y^{\prime}\right) /|y|^{n-1}\right) d y .
$$

Also let:

$$
\mu_{1}(x)=\left(\int_{0}^{\infty} \frac{\left|G_{t}(x)\right|^{2}}{t^{3}} d t\right)^{1 / 2}, \quad \mu_{2}(x)=\left(\int_{0}^{\infty} \frac{\left|H_{t}(x)\right|^{2}}{t^{3}} d t\right)^{1 / 2},
$$

then $F_{t}(x)=G_{t}(x)+H_{t}(x)$; and by Minkowski's inequality:

$$
\mu(f)(x) \leqq \mu_{1}(x)+\mu_{2}(x) .
$$

Define: $\lambda(s)=$ measure of the set where $\mu(f)(x)>s$,

$\lambda_{1}(s)=$ measure of the set where $\mu_{1}(x)>s$,

$\lambda_{2}(s)=$ measure of the set where $\mu_{2}(x)>s$.

Then, because of (3.6) we have $\lambda(s) \leqq \lambda_{1}(s / 2)+\lambda_{2}(s / 2)$. Since we must show that $\lambda(s) \leqq(A / s) \int_{E_{n}} f(x) d x$, it will be sufficient to show that:

$$
\lambda_{i}(s) \leqq(A / s) \int_{E_{n}} f(x) d x, \quad i=1,2 .
$$

Consider $\lambda_{2}(s)$ first. Since $h(x) \in L_{2}\left(E_{n}\right)$, we may apply Lemma 5 ; the result is $\int_{E_{n}}\left(\mu_{2}(x)\right)^{2} d x \leqq A \int_{E_{n}}|h(x)|^{2} d x$.

Thus $\int_{E_{n}}\left(\mu_{2}(x)\right)^{2} d x \leqq A \int_{E_{n}}|h(x)|^{2} d x \leqq A \cdot c \cdot s \int_{E_{n}} f(x) d x$, by (3.4). Now $\lambda_{2}(s) s^{2} \leqq \int_{E_{n}}\left(\mu_{2}(x)\right)^{2} d x \leqq A c s \int_{E_{n}} f(x) d x$. Thus $\lambda_{2}(s) \leqq(A c / s) \int_{E_{n}} f(x) d x$, and (3.7) is proved for $i=2$, with constant $A c$. (The exact constant is of course immaterial for our purposes.)

We next turn to $\mu_{1}(s)$.

Now $\mu_{1}^{2}(x)=\int_{0}^{\infty}\left(\left|G_{t}(x)\right|^{2} / t^{3}\right) d t \leqq \sup _{t>0} \quad\left|G_{t}(x) / t\right| \int_{0}^{\infty}\left(\left|G_{t}(x)\right| / t^{2}\right) d t . \quad$ Call $\int_{0}^{\infty}\left(\left|G_{t}(x)\right| / t^{2}\right) d t=\mu_{0}(x)$. Thus 


$$
\begin{aligned}
\lambda_{1}(s) & =\left|\left\{\mu_{1}(x)>s\right\}\right|=\left|\left\{\mu_{1}^{2}(x)>s^{2}\right\}\right| \\
& \leqq\left|\left\{\sup _{t>0}\left|\frac{G_{t}(x)}{t}\right|>s\right\}\right|+\left|\left\{\mu_{0}(x)>s\right\}\right| .
\end{aligned}
$$

However, by Lemma $6, \sup _{t}\left|G_{t}(x) / t\right| \leqq A g^{*}(x)$. Thus by Lemma 2 , $\left|\left\{\sup _{t>0}\left|G_{t}(x) / t\right|>s\right\}\right| \leqq(A / s) \int|g(x)| d x$ and, therefore,

$$
\left|\left\{\sup _{t>0}\left|G_{t}(x) / t\right|>s\right\}\right| \leqq(A / s) \int_{E_{n}} f(x) d x,
$$

by (3.5).

In order to conclude the estimate $\lambda_{1}(s) \leqq(A / s) \int_{E_{n}} f(x) d x$, we need, therefore, to show that:

$$
\left|\left\{\mu_{0}(x)>s\right\}\right| \leqq(A / s) \int_{E_{n}} f(x) d x .
$$

The proof of (3.8) will take up the rest of the proof of this lemma.

Now, let $\left\{I_{k}\right\}$ be the open cubes whose union is $E_{s}$. Let $x^{k}$ be the center of $I_{k}$, and $d_{k}=$ diameter of $I_{k}$ (i.e. the length of longest diagonal); then $d_{k}=\beta\left|I_{k}\right|^{1 / n}$.

Let $S_{k}$ be the open sphere whose center is $x^{k}$, and whose diameter is $2 d_{k}$. Let $E_{s}^{*}=\bigcup_{k=0} S_{k}$, and $P^{*}$ the closed set complementary to $E_{s}^{*}$. Since $\left|E_{s}\right|$ $=\left|U I_{k}\right| \leqq(1 / s) \int_{E_{n}} f(x) d x$, and all the $I_{k}$ are disjoint, then $\left|E_{s}^{*}\right| \leqq(\gamma / s)$ - $\int_{E_{n}} f(x) d x$. For any $x \in P^{*}$, let $d\left(x, I_{k}\right)=$ distance of $x$ from $I_{k}$; we notice that: $\alpha_{1}\left|x-x^{k}\right| \leqq d\left(x, I_{k}\right)$ as long as $x \in P^{*}$.

It is now our purpose to prove, that if $x \in P^{*}$, then

$$
\mu_{0}(x) \leqq A\left(\sum_{k=0}^{\infty} \frac{\left|I_{k}\right|^{\alpha / n}}{\left|x-x^{k}\right|^{n+\alpha}} \int_{I_{k}}|g(y)| d y\right) .
$$

Now

$$
\mu_{0}(x)=\int_{0}^{\infty} \frac{\left|G_{t}(x)\right|}{t^{2}} d t=\int_{0}^{\infty} \frac{d t}{t^{2}}\left|\int_{\{|x-y| \leqq t\}} g(y) \frac{\Omega(x-y)}{|x-y|^{n-1}} d y\right| .
$$

However, $g(y)$ is nonzero only as long as $y$ lies in some $I_{k}$. Thus,

$$
\begin{aligned}
\mu_{0}(x) & =\int_{0}^{\infty} \frac{d t}{t^{2}}\left|\sum_{k=0}^{\infty} \int_{\left\{I_{k} \cap|x-y| \leqq t\right\}} g(y) \frac{\Omega(x-y)}{|x-y|^{n-1}} d y\right| \\
& \leqq \sum_{k=0}^{\infty} \int_{0}^{\infty} \frac{d t}{t^{2}}\left|\int_{\left\{I_{k} \cap|x-y| \leqq t\right\}} g(y) \frac{\Omega(x-y)}{|x-y|^{n-1}} d y\right| .
\end{aligned}
$$

In order to prove (3.9), it is therefore sufficient to show that:

(13) The $\alpha$ which appears in (3.9) is the same $\alpha$ which is the order of the Lipschitz condition imposed on $\Omega$, by condition (2.5). 


$$
\begin{aligned}
\int_{0}^{\infty} \frac{d t}{t^{2}}\left|\int_{\left\{I_{k} \cap|x-y| \leqq t\right\}} g(y) \frac{\Omega(x-y)}{|x-y|^{n-1}} d y\right| \\
\leqq A \frac{\left|I_{k}\right|^{\alpha / n}}{\left|x-x^{k}\right|^{n+\alpha}} \cdot \int_{I_{k}}|g(y)| d y,
\end{aligned}
$$

where $A$ is independent of $k$.

We now break up the left-hand side of (3.10) as follows:

$$
\begin{aligned}
\int_{0}^{\infty} \frac{d t}{t^{2}}|\cdots|= & \int_{0}^{d\left(x, I_{k}\right)} \frac{d t}{t^{2}}|\cdots|+\int_{d\left(x, I_{k}\right)}^{d\left(x, I_{k}\right)+d_{k}} \frac{d t}{t^{2}}|\cdots| \\
& +\int_{d\left(x, I_{k}\right)+d_{k}}^{\infty} \frac{d t}{t^{2}}|\cdots| \\
= & J_{1}(x)+J_{2}(x)+J_{3}(x) .
\end{aligned}
$$

As far as $J_{1}$ is concerned, we note that the set of $y$ where: $y \in I_{k},|x-y| \leqq t$, and $t<d\left(x, I_{k}\right)$, is empty. Thus $J_{1}=0$. Next consider $J_{2}$.

$$
J_{2}(x)=\int_{d\left(x, I_{k}\right)}^{d\left(x, I_{k}\right)+d_{k}} \frac{d t}{t^{2}}|\cdots| \leqq A \int_{d\left(x, I_{k}\right)}^{d\left(x, I_{k}\right)+d_{k}} \frac{d t}{t^{2}} \int_{I_{k}} \frac{|g(y)|}{|x-y|^{n-1}} d y .
$$

Now if $y \in I_{k}$, then $|x-y| \geqq d\left(x, I_{k}\right)$.

Thus $J_{2}(x) \leqq A d_{k} \cdot\left(d\left(x, I_{k}\right)\right)^{-n-1} \int_{I_{k}}|g(y)| d y$.

We noted before that: $d_{k}=\beta\left|I_{k}\right|^{1 / n}$, and $d\left(x, I_{k}\right)>\alpha_{1}\left|x-x^{k}\right|$. Combining these we get:

$$
J_{2}(x) \leqq A \frac{\left|I_{k}\right|^{1 / n}}{\left|x-x^{k}\right|^{n+1}} \int_{I_{k}}|g(y)| d y \leqq\left(\frac{A\left|I_{k}\right|^{\alpha / n}}{\left|x-x^{k}\right|^{n+\alpha}} \int_{I_{k}}|g(y)| d y\right),
$$

since $\left|I_{k}\right|^{1 / n} \leqq\left|x-x^{k}\right|$, if $x \in P^{*}$, and $0<\alpha \leqq 1$. Finally, if $t>d\left(x, I_{k}\right)+d_{k}$, then $\{|x-y| \leqq t\} \cap I_{k}=I_{k}$. Thus

$$
J_{3}(x)=\int_{d\left(x, I_{k}\right)+d_{k}}^{\infty} \frac{d l}{t^{2}}\left|\int_{I_{k}} g(y) \frac{\Omega(x-y)}{|x-y|^{n-1}} d y\right| .
$$

However,

$$
\int_{I_{k}} g(y) \frac{\Omega(x-y)}{|x-y|^{n-1}} d y=\int_{I_{k}} g(y)\left[\frac{\Omega(x-y)}{|x-y|^{n-1}}-\frac{\Omega\left(x-x^{k}\right)}{\left|x-x^{k}\right|^{n-1}}\right] d y,
$$

since $\int_{I_{k}} g(y) d y=0$. Now,

$$
\begin{aligned}
\left|\frac{\Omega(x-y)}{|x-y|^{n-1}}-\frac{\Omega\left(x-x^{k}\right)}{\left|x-x^{k}\right|^{n-1}}\right| \leqq & \left|\frac{\Omega(x-y)}{|x-y|^{n-1}}-\frac{\Omega(x-y)}{\left|x-x^{k}\right|^{n-1}}\right| \\
& +\left|\frac{\Omega(x-y)-\Omega\left(x-x_{k}\right)}{\left|x-x_{k}\right|^{n-1}}\right| .
\end{aligned}
$$


The first absolute value on the right-hand side is less then

$$
(n-1) \frac{|\Omega(x-y)|\left|y-x_{k}\right|}{\left|x-y_{k}^{*}\right|^{n}},
$$

where $y_{k}^{*}$ is some point in $I_{k}$. Since $x \in P^{*}$, this is less than

$$
\frac{A\left|I_{k}\right|^{1 / n}}{\left|x-x^{k}\right|^{n}} \leqq \frac{A\left|I_{k}\right|^{\alpha / n}}{\left|x-x^{k}\right|^{n-1+\alpha}} .
$$

Moreover,

$$
\left|\frac{\Omega(x-y)-\Omega\left(x-x^{k}\right)}{\left|x-x^{k}\right|^{n-1}}\right| \leqq \frac{A\left|(x-y)^{\prime}-\left(x-x^{k}\right)^{\prime}\right|^{\alpha}}{\left|x-x^{k}\right|^{n-1}},
$$

because of Lipschitz condition on $\Omega$.

However, $\left|(x-y)^{\prime}-\left(x-x^{k}\right)^{\prime}\right| \leqq\left|I_{k}\right|^{1 / n} /\left|x-x^{k}\right|$. Thus combining these estimates we get

$$
\left|\frac{\Omega(x-y)}{|x-y|^{n-1}}-\frac{\Omega\left(x-x^{k}\right)}{\left|x-x^{k}\right|^{n-1}}\right| \leqq \frac{A\left|I_{k}\right|^{\alpha / n}}{\left|x-x^{k}\right|^{n-1+\alpha}}, \quad \text { if } x \in P^{*} .
$$

Therefore

$$
\left|\int_{I_{k}} g(y) \frac{\Omega(x-y)}{|x-y|^{n-1}} d y\right| \leqq \frac{A\left|I_{k}\right|^{\alpha / n}}{\left|x-x^{k}\right|^{n-1+\alpha}} \int_{E_{n}}|g(y)| d y
$$

as long as $x \in P^{*}$. Thus

$$
\begin{aligned}
J_{3}(x) & \leqq \frac{A\left|I_{k}\right|^{\alpha / n}}{\left|x-x^{k}\right|^{n-1+\alpha}}\left(\int_{I_{k}}|g(y)| d y\right) \int_{d\left(x, I_{k}\right)}^{\infty} \frac{d t}{t^{2}} \\
& \leqq \frac{A^{\prime}\left|I_{k}\right|^{\alpha / n}}{\left|x-x^{k}\right|^{n+\alpha}} \int_{I_{k}}|g(y)| d y,
\end{aligned}
$$

since $d\left(x, I_{k}\right)>\alpha_{1}\left|x-x^{k}\right|$. Combining these estimates for $J_{1}, J_{2}$, and $J_{3}$ we prove (3.10). Thus (3.9) is also demonstrated. Therefore

$$
\int_{P^{*}} \mu_{0}(x) d x \leqq A\left(\sum_{k}\left(\int_{P^{*}} \frac{d x}{\left|x-x^{k}\right|^{n+\alpha}} \cdot\left|I_{k}\right|^{\alpha / n} \int_{I_{k}}|g(y)| d y\right)\right) .
$$

But,

$$
\int_{P^{*}} \frac{d x}{\left|x-x^{k}\right|^{n+\alpha}} \leqq \int_{\left|x-x^{k}\right| \geqq 2 d_{k}} \frac{d x}{\left|x-x^{k}\right|^{n+\alpha}} \leqq A\left|I_{k}\right|^{-\alpha / n}
$$

Thus

$$
\int_{P^{*}} \mu_{0}(x) d x \leqq A \sum_{k} \int_{I_{k}}|g(y)| d y=A \int_{E_{n}}|g(y)| d y \leqq A^{\prime} \int_{E_{n}} f(x) d x .
$$


If $E_{s}^{* *}$ is the set in $P^{*}$, where $\mu_{0}(x)>s$ then,

$$
s\left|E_{s}^{* *}\right| \leqq \int_{P^{*}} \mu_{0}(x) d x \leqq A \int_{E_{n}} f(x) d x
$$

Hence,

$$
\left|E_{s}^{* *}\right| \leqq(A / s) \int_{E_{n}} f(x) d x .
$$

However the complement of $P^{*}$ is $E_{s}^{*}$, and $\left|E_{s}^{*}\right| \leqq(\gamma / s) \int_{E_{n}} f(x) d x$. Thus the set $\left\{\mu_{0}(x)>s\right\} \subset E_{s}^{* *} \cup E_{s}^{*}$, and has measure $\leqq(A / s) \int f(x) d x$. This establishes (3.8) and concludes the proof of the lemma.

We are now in a position to state the main result of this section.

Theorem 1. Let $f(x) \in L_{p}\left(E_{n}\right), 1 \leqq p \leqq 2$, and let $\mu(f)=\left(\int_{0}^{\infty}\left(\left|F_{t}(x)\right|^{2} / t^{3}\right) d t\right)^{1 / 2}$, with $F_{t}(x)=\int_{|y| \leq t} f(x-y)\left(\Omega\left(y^{\prime}\right) /|y|^{n-1}\right) d y$, and with $\Omega$ satisfying conditions (2.5) and (2.6) of $\$ 2$.

THEN: $(1) \mu(f)$ is finite almost everywhere,

(2) If $1<p \leqq 2$, then $\|\mu(f)\|_{p} \leqq A_{p}\|f\|_{p}$, and

(3) If $p=1$, then the measure of the set where $\mu(f)(x)>s, s>0$, is less than $(A / s) \int_{E_{n}}|f(x)| d x$.

Proof of the theorem. If $f(x) \in L_{p}\left(E_{n}\right) 1 \leqq p \leqq 2$, write $f(x)=f_{1}(x)+f_{2}(x)$ where $f_{2}(x)=f(x)$ if $|f(x)| \leqq 1$, and $f_{2}(x)=0$ otherwise; then $f_{2}(x) \in L_{2}\left(E_{n}\right)$, and $f_{1}(x) \in L_{1}\left(E_{n}\right)$.

However, $\mu(f) \leqq \mu\left(f_{1}\right)+\mu\left(f_{2}\right) . \mu\left(f_{2}\right)$ is finite a.e. by Lemma 5 , while $\mu\left(f_{1}\right)$ is finite a.e. by Lemma 7. Thus (1) is proved. (3), of course, is the content of Lemma 7.

Finally $\mu(f)$ is an operator satisfying the conditions of the Marcinkiewicz Interpolation Theorem (Lemma 3) with $p_{1}=1, p_{2}=2$. (2) then follows as a result of the Interpolation Lemma. This concludes the proof of Theorem 1 .

4. Marcinkiewicz integral; case when $1<p<\infty$, for odd kernels. In the previous section, we established the results for the generalized Marcinkiewicz integral (when $1 \leqq p \leqq 2$ ) without use of the known one-dimensional case. In this section we shall take the one-dimensional case for granted, and from it establish a result holding for an important class of kernels $\Omega$, for $1<p<\infty$.

To be specific, we shall take the following for granted:

Lemma 8. Let $f(x) \in L_{p}(-\infty,+\infty), 1<p<\infty$ and let $F(x)=\int_{0}^{x} f(t) d t$. DEFINE

$$
\nu(f)=\left(\int_{0}^{\infty} \frac{|F(x+t)+F(x-t)-2 F(x)|^{2}}{t^{3}} d t\right)^{1 / 2} .
$$

THEN : $\|\nu(f)\|_{p} \leqq A_{p}\|f\|_{p}, 1<p<\infty$. 
The periodic analogue of this lemma is due to A. Zygmund [11]. Waterman [10] has found that Zygmund's proof extends to the above nonperiodic case, without essential change. The arguments in either case, however, are far from simple.

Again, we shall assume that $\Omega(x)$ is a function which is homogeneous of degree 0 . In place of condition (2.5) we shall assume that $\Omega\left(x^{\prime}\right)$ is absolutely integrable on $\Sigma$.

We shall also make the following added assumption:

$$
\Omega\left(x^{\prime}\right) \text { is an odd function, i.e. } \Omega\left(x^{\prime}\right)=-\Omega\left(-x^{\prime}\right) \text {. }
$$

From (4.1) and (4.2) it follows immediately that $\int_{\Sigma} \Omega\left(x^{\prime}\right) d \Sigma=0$. This condition was previously assumed.

Our result is then:

Theorem $2\left({ }^{14}\right)$. Assume that $\Omega\left(x^{\prime}\right)$ satisfies conditions (4.1) and (4.2). Let $f(x) \in L_{p}\left(E_{n}\right), 1<p<\infty$.

As before, define $F_{t}(x)=\int_{|y| \leqq t} \Omega\left(y^{\prime}\right) /|y|^{n-1} f(x-y) d y$, and

$$
\mu(f)(x)=\left(\int_{0}^{\infty} \frac{\left|F_{t}(x)\right|^{2}}{t^{3}} d t\right)^{1 / 2} .
$$

Then $\|\mu(f)\|_{p} \leqq A_{p}\|f\|_{p}, 1<p<\infty$.

Proof. The operator $\mu(f)$ is nonlinear. First we introduce a device which allows us to "linearize" the problem. Let $\phi(x, t)$ be a function defined for $x \in E_{n}, 0<t<\infty$, which satisfies the following conditions:

(4.3) $\phi(x, t)$ vanishes if $t$ is small enough, or if $t$ is large enough, and is bounded.

$$
\int_{0}^{\infty} \frac{|\phi(x, t)|^{2}}{t^{3}} d t \leqq 1
$$

for all $x$.

Let $T(f)(x)=\int_{0}^{\infty} F_{t}(x) \phi(x, t) d t / t^{3}$. Because of conditions (4.3) and (4.4), and by Schwarz's inequality: $|T f(x)| \leqq \mu(f)(x)$, and $\mu(f)(x)=\sup _{\phi(x, t)}|T(f)(x)|$, for all $\phi(x, t)$ satisfying (4.3) and (4.4).

Therefore, it will be sufficient to show that

$$
\|T(f)(x)\|_{p} \leqq A_{p}\|f\|_{p},
$$$$
1<p<\infty,
$$

where $A_{p}$ is independent of $\phi(x, t)$ and $f(x)$. Now $\left({ }^{15}\right)$,

(14) This theorem and its proof are inspired by a similar situation that exists in the generalization of the Hilbert transform and which was exploited in [3].

(15) We should point out here that because of the assumptions on $\phi(x, t)$, the interchanges of orders of integration that follow in this proof are all easily justified by Fubini's theorem. 


$$
T f(x)=\int_{0}^{\infty} \frac{F_{t}(x) \phi(x, t) d t}{t^{3}}=\int \frac{\phi(x, t) d t}{t^{3}} \int_{|y| \leq t} f(x-y) \frac{\Omega\left(y^{\prime}\right)}{|y|^{n-1}} d y .
$$

Introduce $r=|y|$, and notice that $d y=r^{n-1} d r d \Sigma$. Then, $T(f)(x)$ $=\int_{0}^{\infty}\left(\phi(x, t) d t / t^{3}\right) \int_{0}^{t} f\left(x-y^{\prime} r\right) \Omega\left(y^{\prime}\right) d r d \Sigma$.

However, $\Omega\left(y^{\prime}\right)=-\Omega\left(-y^{\prime}\right)$, thus

$$
\left.T f(x)=\frac{1}{2} \int_{0}^{\infty} \frac{\phi(x, t) d t}{t^{3}}\left(\int_{0}^{t} f\left(x-y^{\prime} r\right)-f\left(x+y^{\prime} r\right)\right) d r\right) \Omega\left(y^{\prime}\right) d \Sigma .
$$

Fix the direction $y^{\prime}$, and let

$$
\nu_{y^{\prime}}(f)(x)=\int_{0}^{\infty} \frac{\phi(x, t)}{t^{3}}\left(\int_{0}^{t}\left(f\left(x-y^{\prime} r\right)-f\left(x+y^{\prime} r\right)\right) d r\right) d t .
$$

Let $L$ be a line in $E_{n}$, whose direction is parallel to $y^{\prime}$. If $f_{1}(t)$ is the function $f(x)$ restricted to $L$, then

$$
\int_{0}^{t}\left(f\left(x-y^{\prime} r\right)-f\left(x+y^{\prime} r\right)\right) d r=F(x+t)+F(x-t)-2 F(x),
$$

where $F(x)=\int_{0}^{x} f_{1}(t) d t$. Thus using condition (4.4),

$$
\left|\nu_{y^{\prime}}(f)(x)\right| \leqq \nu\left(f_{1}\right)(x), \quad \text { if } x \in L .
$$

Thus, by Lemma 8 ,

$$
\int_{L}\left|\nu_{y^{\prime}}(f)(x)\right|^{p} d x \leqq A_{p}^{p} \int_{L}|f(x)|^{p} d x, \quad 1<p<\infty .
$$

Integrating the above for all lines $L$ whose direction is $y^{\prime}$, results in

$$
\int_{E_{n}}\left|\nu_{y^{\prime}}(f)(x)\right|^{p} d x \leqq A_{p}^{p} \int_{E_{n}}|f(x)|^{p} d x .
$$

Thus,

$$
\left\|\nu_{y^{\prime}}(f)(x)\right\|_{p} \leqq A_{p}\|f\|_{p}
$$

By (4.6), however,

$$
T(f)(x)=2^{-1} \int_{\Sigma} \Omega\left(y^{\prime}\right) \nu_{y^{\prime}}(f)(x) d \Sigma .
$$

Therefore,

$$
\|T f\|_{p} \leqq 2^{-1} A_{p}\left(\int_{\Sigma}|\Omega| d \Sigma\right)\|f\|_{p}
$$

by Minkowski's inequality for integrals. 
This is (4.5), and thus Theorem 2 is proved.

5. The functions $g$ and $S$; case when $1 \leqq p \leqq 2$. Let $f(x) \in L_{p}\left(E_{n}\right), 1 \leqq p$ $<\infty$. Define the "Poisson integral" $U(f)(x ; t)$, for $t>0$, of the function $f(x)$, as follows:

$$
\begin{aligned}
& U(f)(x ; t)=c_{n} \cdot t \int_{E_{n}} \frac{f(x-y) d y}{\left(|y|^{2}+t^{2}\right)^{(n+1) / 2}}, \\
& \text { where } c_{n}=\pi^{-(n+1) / 2} \cdot \Gamma\left(\frac{n+1}{2}\right) .
\end{aligned}
$$

Then $U(f)(x ; t)$ is harmonic in $x_{1}, x_{2}, \cdots, x_{n} ; t, t>0$, and $\lim _{t \rightarrow 0} U(f)(x ; t)$ $=f(x)$ for almost every $x \in E_{n}$, and also in the $L_{p}$ norm.

Moreover $\|U(f)(x ; t)\|_{p} \leqq\|f(x)\|_{p}, 1 \leqq p, t>0$, and $|U(f)(x ; t)| \leqq A t^{-n / p}\|f\|_{p}$, $1 \leqq p, t>0$.

We shall also deal with the so-called "Riesz transforms," $R_{i}(f), i=1, \cdots$, $n$. These are defined by

$$
R_{i}(f)(x)=\lim _{\epsilon \rightarrow 0} c_{n} \cdot \int_{|y|>\epsilon>0} \frac{y_{i}}{|y|^{n+1}} f(x-y) d y .
$$

The $R_{i}(f)$ fall under the class of singular integrals covered by Lemma 4 . For $f(x) \in L_{p}\left(E_{n}\right), 1<p<\infty$, the following identities hold:

$$
\frac{\partial}{\partial t} U\left(R_{i}(f)\right)(x ; t)=\frac{\partial}{\partial x_{i}} U(f)(x ; t), \quad t>0,
$$

and

$$
\frac{\partial}{\partial t} U(f)(x ; t)=-\sum_{i=1}^{n} \frac{\partial}{\partial x_{i}} U\left(R_{i}(f)\right)(x ; t), \quad t>0 .
$$

These may be verified by proving them first for $f \in L_{2}\left(E_{n}\right)$ by the Fourier transform; and then tending to the limit in $L_{p}\left(E_{n}\right)$, by the boundedness of $R_{i}(f)$ in $L_{p}\left({ }^{16}\right)$.

For $f(x) \in L_{p}\left(E_{n}\right)$, and $U(x ; t)=U(f)(x ; t)$, we shall define grad $U$ by

$$
(\operatorname{grad} U(x ; t))^{2}=\left(\frac{\partial U}{\partial t}\right)^{2}+\sum_{i=1}^{n}\left(\frac{\partial U}{\partial x_{i}}\right)^{2} .
$$

As a generalization of the $g$ function of Littlewood and Paley we shall define

$$
g(f)(x)=\left(\int_{0}^{\infty} t \operatorname{grad}^{2} U(x ; t) d t\right)^{1 / 2} .
$$

(16) The reader is referred to [4] where the material in the preceding paragraphs is discussed. The identities (5.3) and (5.4) may be thought of as generalizations of the CauchyRiemann equations. 
The "area integral" of Lusin, (1.2), will be given a similar generalization.

Let $W(x)$ be the set of points in the space $\left(y_{1}, t_{2}, \cdots, y_{n}, t\right), t>0$, which satisfy the condition

$$
|x-y| \leqq \eta t
$$

for some fixed $\eta, 0<\eta<\infty$. Then $W(x)$ is a "cone" whose vertex is $x$.

Define $S(f)(x)$ by:

$$
S(f)(x)=\left(\iint_{W(x)} \frac{(\operatorname{grad} U(y ; t))^{2}}{t^{n-1}} d t d y\right)^{1 / 2} .
$$

(The dependence of $S(f)(x)$ on the parameter $\eta$ will be displayed whenever necessary.)

We first prove a lemma whose one-dimensional periodic analogue is due to Marcinkiewicz and Zygmund [9].

Lemma 9. $g(f)(x) \leqq A_{\eta} S(f)(x)$.

Proof. Since $S(f)(x)$ increases as the parameter $\eta$ increases, we shall assume for the purposes of this lemma that $0<\eta \leqq 1$. Now

$$
\begin{aligned}
g^{2}(f)(x) & =\int_{0}^{\infty} t \operatorname{grad}^{2} U(x ; t) d t=\int_{0}^{1} t \operatorname{grad}^{2} U(x ; t) d t+\int_{1}^{\infty} t \operatorname{grad}^{2} U(x ; t) d t \\
& =I_{1}+I_{2} .
\end{aligned}
$$

But,

$$
\begin{aligned}
I_{1} & =\sum_{k=0}^{\infty} \int_{2^{-k-1}}^{2^{-k}} t \operatorname{grad}^{2} U(x ; t) d t \\
& \leqq \sum_{k=0}^{\infty} 2^{-k} \operatorname{grad}^{2} U\left(x ; r_{k}\right)
\end{aligned}
$$

where $r_{k}$ is a value of $t$ in the interval $\left[2^{-k-1}, 2^{-k}\right]$ at which $\operatorname{grad}^{2} U(x ; t)$ attains its maximum.

Since $U(x ; t)$ is harmonic in $x_{1} \cdots x_{n}, t$, then $\operatorname{grad}^{2} U(x ; t)$ is subharmonic there. Thus,

$$
\operatorname{grad}^{2} U\left(x ; r_{k}\right) \leqq \frac{1}{\left|S_{k}\right|} \int_{S_{k}} \operatorname{grad}^{2} U(y ; t) d y d t .
$$

Here $S_{k}$ is the sphere in the $n+1$ dimensional space $y_{1} \cdots y_{n}, t$ whose center is $\left(x, r_{k}\right)$ and whose diameter is $\eta 2^{-k-1} ;\left|S_{k}\right|$ denotes the $(n+1)$ dimensional volume of the sphere $S_{k}$. It is easily observed that $S_{k} \subset W(x)$. Moreover, the $S_{k}$ with even $k$ are all disjoint; similarly the $S_{k}$ with odd $k$ are all disjoint.

Now $\left|S_{k}\right|=\alpha \cdot \eta^{n} 2^{-n k-n}$, where $\alpha$ depends only on $n$. 
Therefore,

$$
\begin{aligned}
\operatorname{grad}^{2} U\left(x ; r_{k}\right) & \leqq\left(1 / \alpha \cdot \eta^{n}\right) \cdot 2^{n k+n} \int_{S_{k}} \operatorname{grad}^{2} U(y ; t) d t d y \\
& \leqq\left(1 / \alpha \cdot \eta^{n}\right) 2^{n k+n} 2^{-(n-1)(k-1)} \int_{S_{k}} \frac{\operatorname{grad}^{2} U(y ; t) d t d y}{t^{n-1}}
\end{aligned}
$$

since $t \geqq 2^{-k-1}$ in $S_{k}$. Hence,

$$
\begin{aligned}
\sum_{k \text { even }} 2^{-k} \operatorname{grad}^{2} U\left(x ; r_{k}\right) & \leqq\left(1 / \alpha \cdot \eta^{n}\right) \cdot 2^{2 n+1} \sum_{k \text { even }} \int_{S_{k}} \frac{\operatorname{grad}^{2} U(y ; t) d t d y}{t^{n-1}} \\
& \leqq A_{\eta} S^{2}(f)(x) .
\end{aligned}
$$

Similarly, $\sum_{k \text { odd }} 2^{-k} \operatorname{grad} U^{2}\left(x ; r_{k}\right) \leqq A_{\eta} S^{2}(f)(x)$.

This proves that $I_{1} \leqq A_{\eta} S^{2}(f)(x)$. In proving that $I_{2} \leqq A_{\eta} S^{2}(f)(x)$, we break up the integral $(1, \infty)$ into intervals of the form $\left[2^{k}, 2^{k+1}\right]$ and proceed as before. This completes the proof of Lemma 9.

Lemma 10. If $f(x) \in L_{2}\left(E_{n}\right)$, then $S(f)(x) \in L_{2}\left(E_{n}\right)$ and

$$
\|S(f)(x)\|_{2} \leqq A_{\eta}\|f(x)\|_{2} .
$$

Proof. Let $\psi_{t}(y)$ be the characteristic function of the set $|y| \leqq \eta t$. Then

$$
S^{2}(f)(x)=\int_{0}^{\infty} \int_{E_{n}} \frac{\psi_{t}(y)}{t^{n-1}} \operatorname{grad}^{2} U(x-y ; t) d y d t .
$$

If $\hat{f}(x)$ denotes the Fourier transform of $f(x)$, then by Fubini's and Plancherel's theorems:

$$
\begin{aligned}
\int_{E_{n}} S^{2}(f)(x) d x & =\int_{0}^{\infty} \frac{d t}{t^{n-1}} \int_{E_{n}} \psi_{t}(y) d y \int_{E_{n}} \operatorname{grad}^{2} U(x-y ; t) d x \\
& =2 \cdot(2 \pi)^{n} \int_{0}^{\infty} \frac{d t}{t^{n-1}} \int_{E_{n}} \psi_{t}(y) d y \int_{E_{n}}|x|^{2} e^{-2|x| t \mid}|\hat{f}(x)|^{2} d x \\
& =2 \cdot(2 \pi)^{n} d_{n} \eta^{n} \int_{0}^{\infty} t d t \int_{E_{n}}|x|^{2} e^{-21 x \mid t}|\hat{f}(x)|^{2} d x .
\end{aligned}
$$

Since $\int_{E_{n}} \psi_{t}(y) d y=\int_{|y| \leq \eta t} d y=d_{n} \eta^{n} t^{n}$, where $d_{n}$ is the volume of the unit sphere in $n$-space. Thus,

$$
\begin{aligned}
\int_{E_{n}} S^{2}(f)(x) d x & =2 \cdot(2 \pi \eta)^{n} d_{n} \int_{E_{n}}|x|^{2}|\hat{f}(x)|^{2} d x \int_{0}^{\infty} t e^{-2 t|x|} d t \\
& =2 \cdot(2 \pi \eta)^{n} \cdot d_{n} \cdot 1 / 4 \int_{E_{n}}|\hat{f}(x)|^{2} d x, \text { because }
\end{aligned}
$$




$$
\int_{0}^{\infty} t e^{-2 t|x|} d t=\frac{1}{4|x|^{2}}
$$

Using Plancherel's theorem again we thus get

$$
\int_{E_{n}} S^{2}(f)(x) d x=1 / 2(\eta)^{n} \cdot d_{n} \cdot \int_{E_{n}}|f(x)|^{2} d x
$$

and the lemma is proved.

Lemma 11. Let $f(x) \in L_{p}\left(E_{n}\right), 1 \leqq p \leqq \infty$, and let $U(x ; t)$ be its Poisson integral, as defined by (5.1). For any fixed $\eta, 0<\eta<\infty$, let $W(x)$ denote the set of points $y$ so that $|x-y| \leqq \eta t$. Then

$$
\sup _{y \in W(x)}\left|\frac{t \cdot \partial U(y ; t)}{\partial t}\right| \leqq A_{\eta} f^{*}(x) .
$$

Similarly,

$$
\sup _{y \in W(x)}\left|\frac{t \cdot \partial U(y ; t)}{\partial x_{i}}\right| \leqq A_{\eta}{ }^{*}(x), \quad i=1, \cdots, n .
$$

$f^{*}(x)$ is the "maximal" function defined in Lemma 2.

Proof.

$$
\frac{\partial U(x ; t)}{\partial x_{i}}=\frac{-(n+1)}{2} c_{n} \cdot t \cdot \int \frac{y_{i} \cdot f(x-y)}{\left(|y|^{2}+t^{2}\right)^{(n+3) / 2}} d y .
$$

Thus

$$
\begin{aligned}
& \left|\frac{t \partial U(x+z, t)}{\partial x_{i}}\right|=\left(\frac{n+1}{2}\right) c_{n} \cdot t^{2} \cdot\left|\int \frac{\left(y_{i}-z_{i}\right)}{\left(|y-z|^{2}+t^{2}\right)^{(n+3) / 2}} f(x-y) d y\right| \\
& \quad \leqq A t^{2} \int_{|y| \leqq 2 \eta t} \frac{\left|y_{i}-z_{i}\right||f(x-y)| d y}{\left(|y-z|^{2}+t^{2}\right)^{(n+3) / 2}}+\int_{|y|>2 \eta t} \frac{\left|y_{i}-z_{i}\right||f(x-y)| d y}{\left(|y-z|^{2}+t^{2}\right)^{(n+3) / 2}} \\
& \quad=I_{1}+I_{2} .
\end{aligned}
$$

Now $I_{1} \leqq A t^{2} \int_{|y| \leqq 2 \eta t} 3 \eta t|f(x-y)| d y /\left(t^{2}\right)^{(n+3) / 2}$, since $|z| \leqq \eta t$. Thus

$$
\begin{aligned}
I_{1} & \leqq 3 A \eta \cdot 1 / t^{n} \int_{|y| \leqq 2 \eta t}|f(x-y)| d y \\
& \leqq \cdot A \cdot \eta \cdot(2 \eta)^{n} f^{*}(x) \leqq A_{\eta} f^{*}(x) .
\end{aligned}
$$

Now if $|y|>2 \eta t$, and $|z| \leqq \eta t$, then $|y-z| \geqq|y| / 2$ and $\left|y_{i}-z_{i}\right| \leqq 2|y|$.

Thus $I_{2} \leqq A t^{2} \int_{|y| z 2 \eta t}|f(x-y)| d y /|y|^{n+2}$. However, 


$$
\begin{aligned}
t^{2} \int_{|y| \geqq 2 \eta t} \frac{|f(x-y)| d y}{|y|^{n+2}} & =t^{2} \sum_{k=0}^{\infty} \int_{2^{k+2} \eta t>|y| \geq 2^{k+1} \eta t} \frac{|f(x-y)| d y}{|y|^{n+2}} \\
& \leqq t^{2} \sum_{k=0}^{\infty}\left(2^{k+1} \eta t\right)^{-n-2} \cdot \int_{|y|<2^{(k+2)} \eta t}|f(x-y)| d y \\
& \leqq t^{2} \sum_{k=0}^{\infty}\left(2^{k+1} \eta t\right)^{-n-2} \cdot\left(2^{k+2} \eta t\right)^{n} \cdot A \cdot f^{*}(x) \\
& \leqq A \cdot \eta^{-2}\left(\sum_{k=0}^{\infty} 2^{-2 k+n-2}\right) f^{*}(x) \leqq A_{\eta} f^{*}(x) .
\end{aligned}
$$

Collecting these estimates proves that:

$$
\sup _{t>0,|z| \leqq \eta t}\left|\frac{t \cdot \partial U(x+z ; t)}{\partial x_{i}}\right| \leqq A_{\eta} f^{*}(x),
$$

which establishes the second part of the lemma. The estimate for the first part of the lemma is made along very similar lines.

The following lemma is analogous to Lemma 7. Its proof is closely parallel with the proof of that lemma, so that we shall be correspondingly brief in proving Lemma 12.

Lemma 12. Let $f(x) \in L_{1}\left(E_{n}\right)$. Define $S(f)(x)$ as in (5.6). Then $S(f)(x)$ is finite for almost every $x \in E_{n}$. Moreover, if $s>0$, and $D_{s}$ is the set where $S(f)(x)$ $>s$, then

$$
\left|D_{s}\right| \leqq\left(A_{\eta} / s\right) \int_{E_{n}}|f(x)| d x .
$$

Proof. Assume $f(x) \geqq 0$. Given $s>0$, let $f(x)=g(x)+h(x)$ be the decomposition given by Lemma 1 , and $E_{s}$ the corresponding open set which contains the support of $g(x)$. As in Lemma 7

$$
\int_{E_{n}}|h(x)|^{2} d x \leqq c s \int_{E_{n}} f(x) d x, \text { and } \int_{E_{n}}|g(x)| d x \leqq c \int_{E_{n}} f(x) d x .
$$

Let $U(x ; t), V(x ; t)$ and $W(x ; t)$ be the Poisson integrals of $f(x), g(x)$, and $h(x)$ respectively. Write

$$
\begin{aligned}
S(f)(x) & =\left(\iint_{W(x)} \frac{(\operatorname{grad} U(y ; t))^{2}}{t^{n-1}} d t d y\right)^{1 / 2}, \\
T(x) & =\left(\iint_{W(x)} \frac{(\operatorname{grad} V(y ; t))^{2}}{t^{n-1}} d t d y\right)^{1 / 2}
\end{aligned}
$$

and 


$$
R(x)=\left(\iint_{W(x)} \frac{(\operatorname{grad} W(y ; t))^{2}}{t^{n-1}} d t d y\right)^{1 / 2} .
$$

Since $U(x ; t)=V(x ; t)+W(x ; t)$, then $S(f)(x) \leqq T(x)+R(x)$. If $\lambda(s)$ denotes the measure of the set where $S(f)(x)>s, \lambda_{1}(s)$ the measure of the set where $T(x)>s$, and $\lambda_{2}(s)$ the measure of the set where $R(x)>s$, then

$$
\lambda(s) \leqq \lambda_{1}(s / 2)+\lambda_{2}(s / 2) .
$$

As in the proof of Lemma 7,

$$
\lambda_{2}(s) \leqq\left(A_{\eta} / s\right) \int_{E_{n}} f(x) d x,
$$

since $\int_{E_{n}} R^{2}(x) d x \leqq A_{\eta} \int|h(x)|^{2} d x \leqq A_{\eta} c \cdot s \cdot \int_{E_{n}} f(x) d x$, by Lemma 10 .

It is therefore sufficient to show that

$$
\lambda_{1}(s) \leqq\left(A_{\eta} / s\right) \int_{E_{n}} f(x) d x
$$

However

$$
\begin{aligned}
T^{2}(x) & =\iint_{W(x)} \frac{\operatorname{grad}^{2} V(y ; t)}{t^{n-1}} d t d y \\
& \leqq \sup _{t>0,|z| \leqq \eta t}|t \cdot \operatorname{grad} V(x+z ; t)| \iint_{W(x)} \frac{|\operatorname{grad} V(y ; t)|}{t^{n}} d t d y .
\end{aligned}
$$

Call,

$$
\iint_{W(x)} \frac{|\operatorname{grad} V(y ; t)|}{t^{n}} d t d y=T_{0}(x) .
$$

Then by Lemma 11 ,

$$
T^{2}(x) \leqq A_{\eta} g^{*}(x) T_{0}(x) .
$$

However, the set where $T^{2}(x)>s^{2}$, is included in the union of the set where $A_{\eta} g^{*}(x)>s$, and the set where $T_{0}(x)>s$. But by Lemma 2 , the first set has measure less than $\left(A_{\eta} / s\right) \cdot \int_{E_{n}}|g(x)| d x \leqq\left(A_{\eta} / s\right) \cdot c \int_{E_{n}} f(x) d x$.

Thus, if $\lambda_{0}(s)$ denotes the measure of the set where $T_{0}(x)>s$, we must show that

$$
\lambda_{0}(s) \leqq\left(A_{\eta} / s\right) \int_{E_{n}} f(x) d x .
$$

As before, $I_{k}$ are the open cubes whose union is $E_{8}$. We denote by $x^{k}$ the center of $I_{k}$, and $S_{k}$ the open sphere whose center is $x^{k}$ and whose diameter is twice the diameter of $I_{k}$. If $E_{s}^{*}=U S_{k}$, then $\left|E_{s}^{*}\right| \leqq(\gamma / s) \int_{E_{n}} f(x) d x$. We let $P^{*}=$ complement of $E_{s}^{*}$. 
In analogy with Lemma 7 , it is now our purpose to show that if $x \in P^{*}$

$$
T_{0}(x) \leqq A\left(\sum_{k=1}^{\infty}\left(\frac{\left|I_{k}\right|^{1 / n}}{\left|x-x^{k}\right|^{n+1}} \int_{I_{k}}|g(y)| d y\right) .\right.
$$

For this purpose, it is clearly sufficient to establish

$$
\begin{array}{r}
\int_{0}^{\infty} \frac{d t}{t^{n}} \int_{|z| \leqq \eta t}\left|\frac{\partial V}{\partial x_{i}}(x+z ; t)\right| d z \leqq A_{\eta} \sum_{k}\left(\frac{\left|I_{k}\right|^{1 / n}}{\left|x-x^{k}\right|^{n-1}} \int_{I_{k}}|g(y)| d y\right) \\
\text { for } i=1,2, \cdots n
\end{array}
$$

and

(5.10*) $\int_{0}^{\infty} \frac{d t}{t^{n}} \int_{|z| \leqq \eta t}\left|\frac{\partial V}{\partial t}(x+z ; t)\right| d z \leqq A_{\eta} \sum_{k}\left(\frac{\left|I_{k}\right|^{1 / n}}{\left|x-x^{k}\right|^{n+1}} \int_{I_{k}}|g(y)| d y\right)$,

whenever $x \in P^{*}$.

Let us first deal with (5.10), keeping in mind that $g(x)$ is nonzero only on each $I_{k}$.

$$
\begin{aligned}
\int_{0}^{\infty} \frac{d t}{t^{n}} \int_{|z| \leqq \eta t} \mid & \frac{\partial V}{\partial x_{i}}(x+z ; t) \mid d z \\
& =\int_{0}^{\infty} \frac{d t}{t^{n}} \int_{|z| \leqq \eta t}\left|c \cdot t \int \frac{g(y)\left(x_{i}+z_{i}-y_{i}\right)}{\left(|x+z-y|^{2}+t^{2}\right)^{(n+3) / 2}} d y\right| d z \\
& \leqq c \int_{0}^{\infty} \frac{d t}{t^{n-1}} \int_{|z| \leqq \eta t} d z \sum_{k=1}^{\infty}\left|\int_{I_{k}} \frac{g(y)\left(x_{i}+z_{i}-y_{i}\right)}{\left(|x+z-y|^{2}+t^{2}\right)^{(n+3) / 2}} d y\right| .
\end{aligned}
$$

It will therefore be sufficient to show that

$$
\begin{array}{r}
\int_{0}^{\infty} \frac{d t}{t^{n-1}} \int_{|z| \leq \eta t} d z\left|\int_{I_{k}} \frac{g(y)\left(x_{i}+z_{i}-y_{i}\right)}{\left(|x+z-y|^{2}+t^{2}\right)^{(n+3) / 2}} d y\right| \\
\leqq A_{\eta} \cdot \frac{\left|I_{k}\right|^{1 / n}}{\left|x-x^{k}\right|^{n+1}} \cdot \int_{I_{k}}|g(y)| d y .
\end{array}
$$

Now

$$
\begin{aligned}
& \int_{I_{k}} \frac{g(y)\left(x_{i}+z_{i}-y_{i}\right)}{\left(|x+z-y|^{2}+t^{2}\right)^{(n+3) / 2}} d y \\
& \quad=\int_{I_{k}} g(y)\left[\frac{\left(x_{i}+z_{i}-y_{i}\right)}{\left(|x+z-y|^{2}+t^{2}\right)^{(n+3) / 2}}-\frac{\left(x_{i}+z_{i}-x_{i}^{k}\right)}{\left(\left|x+z-x^{k}\right|^{2}+t^{2}\right)^{(n+3) / 2}}\right] d y,
\end{aligned}
$$

since $\int_{I_{k}} g(y) d y=0$.

A straightforward calculation shows that if $x \in P^{*}, y \in I_{k}, t \leqq(1 / 2 \eta)$ $\cdot\left|x-x^{k}\right|$, and $|z| \leqq \eta t$, then 


$$
\begin{aligned}
\delta & \equiv\left|\frac{\left(x_{i}+z_{i}-y_{i}\right)}{\left(|x+z-y|^{2}+t^{2}\right)^{(n+3) / 2}}-\frac{\left(x_{i}+z_{i}-x_{i}^{k}\right)}{\left(\left|x+z-x^{k}\right|^{2}+t^{2}\right)^{(n+3) / 2}}\right| \\
& \leqq C_{\eta} \frac{\left|I_{k}\right|^{1 / n}}{\left|x-x_{k}\right|^{n+3}} \cdot
\end{aligned}
$$

While if $x \in P^{*}, y \in I_{k},\left|x-x^{k}\right| \leqq 2 \eta t$ and $|z| \leqq \eta t$, then

$$
\delta \leqq C_{\eta} \frac{\left|I_{k}\right|^{1 / n}}{t^{n+3}} .
$$

Thus,

$$
\begin{aligned}
\int_{0}^{\infty} \frac{d t}{t^{n-1}} \int_{|z| \leqq \eta t} d z \mid & \frac{g(y)\left(x_{i}+z_{i}-y_{i}\right)}{I_{I_{k}}} d y \mid \\
& \leqq \int_{0}^{\infty} \frac{d t}{t^{n-1}} \int_{|z| \leqq \eta t} d z \cdot \delta \cdot\left(\int_{I_{k}}|g(y)| d y\right) \\
& =\int_{0}^{(1 / 2 \eta)\left|x-x^{k}\right|} \frac{d t}{t^{n-1}} \cdot \int_{|z| \leqq \eta t} d z \cdot \delta \cdot \int_{I_{k}}|g(y)| d y \\
& +\int_{(1 / 2 \eta)\left|x-x^{k}\right|}^{\infty} \frac{d t}{t^{n-1}} \int_{|z| \leqq \eta t} d z \cdot \delta \cdot \int_{I_{k}}|g(y)| d y \\
& \leqq C_{\eta} \int_{0}^{(1 / 2 \eta)\left|x-x^{k}\right|} \frac{d t}{t^{n-1}} \cdot(\eta t)^{n} \cdot \frac{\left|I_{k}\right|^{1 / n}}{\left|x-x^{k}\right|^{n+3}} \cdot \int_{I_{k}}|g(y)| d y \\
& +C_{\eta} \int_{(1 / 2 \eta)\left|x-x^{k}\right|}^{\infty} \frac{d t}{t^{n-1}} \cdot(\eta t)^{n} \cdot \frac{\left|I_{k}\right|^{1 / n}}{t^{n+3}} \cdot \int_{I_{k}}|g(y)| d y \\
& \leqq C_{\eta} \cdot \frac{\left|I_{k}\right|^{1 / n}}{\left|x-x^{k}\right|^{n+1}} \cdot \int_{I_{k}}|g(y)| d y .
\end{aligned}
$$

Thus (5.11) is demonstrated. This immediately implies (5.10). Using very similar estimates we can prove $\left(5.10^{*}\right)$. Together these imply (5.9).

Because of (5.9), and arguing as in Lemma 7,

$$
\int_{P^{*}} T_{0}(x) d x \leqq A_{\eta} \int_{E_{n}} f(x) d x .
$$

Thus if $E_{s}^{* *}$ is the subset of $P^{*}$ where $T_{0}(x)>s$, then $s\left|E_{s}^{* *}\right| \leqq \int_{P^{*}} T_{0}(x) d x$ $\leqq A_{\eta} \int_{E_{n}} f(x) d x$. Therefore $\left|E^{* *}\right| \leqq\left(A_{\eta} / s\right) \int_{E_{n}} f(x) d x$. However the complement of $P^{*}$ is $E_{s}^{*}$, and $\left|E_{s}^{*}\right| \leqq(\gamma / s) \int_{E_{n}} f(x) d x$. This implies that the measure of the set where $T_{0}(x)>s$ is less than $\left(\left(A_{\eta}+\gamma\right) / s\right) \int_{E_{n}} f(x) d x$.

With this (5.8) is demonstrated, and the proof of the lemma is concluded.

Theorem 3. Let $f(x) \in L_{p}\left(E_{n}\right) 1 \leqq p \leqq 2$. Let $0<\eta<\infty, \eta$ fixed, and let 
$S(f)(x)$ be defined by (5.6). Then (1) $S(f)(x)$ is finite almost everywhere; (2) $\|S(f)(x)\|_{p} \leqq A_{p, \eta}\|f(x)\|_{p}$, if $1<p \leqq 2$ and (3) if $f \in L_{1}\left(E_{n}\right)$, then the measure of the set where $S(f)(x)>s, s>0$, is less than

$$
\left(A_{\eta} / s\right) \int_{E_{n}}|f(x)| d x .
$$

Proof. If $f(x) \in L_{p}\left(E_{n}\right)$, write $f(x)=f_{1}(x)+f_{2}(x)$ where $f_{2}(x)=f(x)$ if $|f(x)| \leqq 1, f_{2}(x)=0$ otherwise. Then $f_{2}(x) \in L_{2}\left(E_{n}\right)$, and $f_{1}(x) \in L_{1}\left(E_{n}\right)$. However, $S(f)(x) \leqq S\left(f_{1}\right)(x)+S\left(f_{2}\right)(x)$. $S\left(f_{2}\right)(x)$ is finite almost everywhere by Lemma 10 , and $S\left(f_{1}\right)(x)$ is finite almost everywhere by Lemma 12 . This proves (1). (3) is contained in the statement of Lemma 12.

$S(f)$ is an operator satisfying the conditions of Lemma 3 with $p_{2}=2$, $p_{1}=1$. Thus as a result of Lemma $3,\|S(f)\|_{p} \leqq A_{p, \eta}\|f\|_{p}$ if $1<p<2$. Combining this with Lemma 10, concludes the proof of the theorem.

CoRollary. If $f(x) \in L_{p}\left(E_{n}\right), 1 \leqq p \leqq 2$, and $g(f)$ is the operator defined by (5.5), then the conclusions of Theorem 3 hold with $S(f)(x)$ replaced by $g(f)(x)$.

Proof. The corollary follows from Theorem 3 by applying Lemma 9.

6. The functions $g$ and $S$; case when $2 \leqq p<\infty$.

Lemma 13. Let $f(x) \in L_{p}\left(E_{n}\right), 1 \leqq p \leqq \infty$, and let $U(x ; t)$ be its Poisson integral as defined by (5.1). Then $\sup _{t>0}|U(x ; t)| \leqq A f^{*}(x)$, where $f^{*}(x)$ is the "maximal" function defined in Lemma 2.

Proof.

$$
\begin{aligned}
L(x ; t)= & c_{n} t \int_{E_{n}} \frac{f(x-y)}{\left(|y|^{2}+t^{2}\right)^{(n+1) / 2}} d y=c_{n} t \int_{|y| \leqslant t} \frac{f(x-y)}{\left(|y|^{2}+t^{2}\right)^{(n+1) / 2}} d y \\
& +c_{n} \cdot t \cdot \int_{|y|>t} \frac{f(x-y)}{\left(|y|^{2}+t^{2}\right)^{(n+1) / 2}} d y .
\end{aligned}
$$

Thus,

$$
|U(x, t)| \leqq A t^{-n} \cdot \int_{|y| \leqslant t}|f(x-y)| d y+A \cdot t \cdot \int_{|y|>t} \frac{|f(x-y)|}{|y|^{n+1}} d y .
$$

The first integral is majorized by $A f^{*}(x)$. In order to complete the proof of the lemma we consider the following:

$$
\begin{aligned}
t \int_{|y|>t} \frac{|f(x-y)|}{|y|^{n+1}} d y & =t \cdot \sum_{k=0}^{\infty} \int_{2^{(k+1)} t \geqq|y|>2^{k} t} \frac{|f(x-y)|}{|y|^{n+1}} d y \\
& \leqq \sum_{k=0}^{\infty} t \cdot 2^{-k(n+1)} t^{-n-1} \cdot \int_{2^{(k+1)} t \geqq|y|}|f(x-y)| d y \\
& \leqq \sum_{k=0}^{\infty} t^{-n} \cdot 2^{-k(n+1)} \cdot 2^{(k+1) n} \cdot t^{n} \cdot f^{*}(x) \leqq A f^{*}(x) .
\end{aligned}
$$


Thus the lemma is proved.

Theorem 4. Let $f(x) \in L_{p}\left(E_{n}\right), 1<p<\infty$, choose a fixed $\eta, 0<\eta<\infty$, and let $S(f)(x)$ be defined by (5.6). Then $\|S(f)(x)\|_{p} \leqq A_{\eta, p}\|f(x)\|_{p}$, if $1<p<\infty$.

Proof $\left({ }^{17}\right)$. Assume first that $p \geqq 4$. Let $q$ be the index conjugate to $p / 2$, i.e. $1 / q+2 / p=1$; thus $1<q \leqq 2$. Then,

$$
\begin{aligned}
\|S(f)(x)\|_{p}^{2} & =\left(\int_{E_{n}}\left(S(f)^{2}(x)\right)^{p / 2} d x\right)^{2 / p} \\
& =\sup _{\phi(x)} \int_{E_{n}} S(f)^{2}(x) \phi(x) d x
\end{aligned}
$$

taken over the class of all $\phi(x)$, so that $\phi(x) \geqq 0, \phi(x)$ is indefinitely differentiable and has compact support, and

$$
\int_{E_{n}}(\phi(x))^{q} d x \leqq 1
$$

Now define $J$ by

$$
J=\int_{E_{n}} S^{2}(f)(x) \phi(x) d x
$$

Thus $J=\iiint \operatorname{grad}^{2} U(y ; t) \psi_{t}(x-y) / t^{n-1} \phi(x) d y d t d x$, where $\psi_{\iota}(y)$ is the characteristic function of the set $|y| \leqq \eta t$. Let $P(y ; t)$ denote the kernel of the Poisson integral (5.1),

$$
P(y ; t)=\frac{c_{n} \cdot t}{\left(|y|^{2}+t^{2}\right)^{(n+1) / 2}} .
$$

Then if $|y| \leqq \eta t, P(y ; t) \geqq t^{-n} / A_{\eta}$. Thus,

$$
J \leqq A_{\eta} \iiint \operatorname{grad} U^{2}(y ; t) P(x-y ; t) \phi(x) d x d y t d t ;
$$

and if $V(x ; t)$ is the Poisson integral of $\phi(x)$, then

$$
J \leqq A_{\eta} \iint \operatorname{grad}^{2} U(y ; t) V(y ; t) t d t d y .
$$

If $\Delta$ denotes the Laplace operator

$$
\Delta=\frac{\partial^{2}}{\partial t^{2}}+\sum_{i=1}^{n} \frac{\partial^{2}}{\partial x_{i}^{2}},
$$

then a simple calculation yields that,

(17) The proof of this theorem is an extension of an argument found in [12]. 


$$
2 \operatorname{grad}^{2} U(x ; t)=\Delta\left(U^{2}(x ; t)\right) .
$$

Moreover, if $a(x ; t), b(x ; t)$ denote arbitrary twice differentiable functions, we easily verify that

$$
\Delta(a b)=a \Delta(b)+b \Delta(a)+2 a_{t} b_{t}+2 \sum_{i=1}^{n} a_{x_{i}} b_{x_{i}} .
$$

Let now $0<\epsilon<L<\infty$, and define $J_{\epsilon, L}$ by

$$
J_{\epsilon, L}=\int_{E_{n}} d y \int_{\epsilon}^{L} t d t \operatorname{grad}^{2} U(y ; t) V(y ; t) .
$$

Apply formula (6.3) where $a=U^{2}(y ; t), b=V(y ; t)$ to $(6.4)$. Then since $\Delta(V(x ; t))=0$, and $\Delta\left(U^{2}(x ; t)\right)=2 \operatorname{grad}^{2} U(x ; t)$, (by (6.2)), we have:

$$
\begin{aligned}
J_{\epsilon, L}= & \frac{1}{2} \int_{E_{n}} d y \int_{\epsilon}^{L} t d l \Delta\left(U^{2} V\right)-2 \int_{E_{n}} d y \int_{\epsilon}^{L} t d t U \cdot U_{t} \cdot V_{t} \\
& -\sum_{i=1}^{n} 2 \int_{E_{n}} d y \int_{\epsilon}^{L} t d t U \cdot U_{y_{i}} \cdot V_{y_{i}} \\
= & I_{1}+I_{2}+I_{3} \text { say. }
\end{aligned}
$$

However,

$$
\begin{aligned}
\left|I_{2}\right| & \leqq 2 \int_{E_{n}} d y \int_{0}^{\infty} t d t\left|U(y ; t) U_{t}(y ; t) V_{t}(y ; t)\right| d t \\
& \leqq A \int_{E_{n}} f^{*}(y) \int_{0}^{\infty} t\left|U_{t}(y ; t) V_{t}(y ; t)\right| d t \leqq A \int_{E_{n}} f^{*}(y) g(f)(y) g(\phi)(y) d y .
\end{aligned}
$$

The next-to-the last step is by Lemma 13, and the last step is by Schwarz's inequality. However,

$$
\begin{aligned}
\int_{E_{n}} f^{*}(y) g(f)(y) g(\phi) & (y) d y \\
& \leqq\left(\int\left(f^{*}(y)\right)^{p} d y\right)^{1 / p}\left(\int(g(f)(y))^{p} d y\right)^{1 / p}\left(\int g(\phi)(y)^{q} d y\right)^{1 / q}
\end{aligned}
$$

by Hölder's inequality, since $2 / p+1 / q=1$.

By Theorem 3, Corollary, we have,

$$
\left(\int(g(\phi)(y))^{q} d y\right)^{1 / q} \leqq A_{q}\|\phi\|_{q} \leqq A_{q},
$$

since $1<q \leqq 2$. Moreover, by Lemma $9, g(f)(y) \leqq A_{\eta} S(f)(y)$; and by Lemma 2 ,

$\left.{ }^{18}\right)$ In this context, letter subscripts denote partial differentiation. 


$$
\left(\int\left(f^{*}(y)\right)^{p} d y\right)^{1 / p} \leqq A_{p}\left(\int|f(y)|^{p} d y\right)^{1 / p}
$$

Combining these estimates we get,

$$
\left|I_{2}\right| \leqq A_{\eta, p}\|f\|_{p}\|S(f)\|_{p}
$$

Similarly,

$$
\left|I_{3}\right| \leqq A_{\eta, p}\|f\|_{p}\|S(f)\|_{p}
$$

Now,

$$
\begin{aligned}
I_{1}= & \frac{1}{2} \int_{E_{n}} d y \int_{\epsilon}^{L} t d t \Delta\left(U^{2} V\right)=\frac{1}{2} \int_{E_{n}} d y \int_{\epsilon}^{L} t \frac{\partial^{2}}{\partial t^{2}}\left(U^{2}(y ; t) V(y ; t)\right) \\
& +\frac{1}{2} \sum_{i=1}^{n} \int_{\epsilon}^{L} t d t \int_{E_{n}} \frac{\partial^{2}}{\partial y_{i}^{2}}\left(U^{2}(y ; t) V(y ; t)\right) d y .
\end{aligned}
$$

We notice first that: $\int_{E_{n}}\left(\partial^{2} / \partial y_{i}^{2}\right)\left(U^{2}(y ; t) V(y ; t)\right) d y=0$. Moreover, by integrating by parts:

$$
\begin{aligned}
\int_{\epsilon}^{L} t \frac{\partial^{2}}{\partial t^{2}}\left(U^{2}(y ; t) V(y ; t)\right)= & \left.2 t U(y ; t) U_{t}(y ; t) V(y ; t)\right]_{\epsilon}^{L} \\
& \left.\left.+t U^{2}(y ; t) V_{t}(y ; t)\right]_{\epsilon}^{L}-U^{2}(y ; t) V(y ; t)\right]_{\epsilon}^{L} .
\end{aligned}
$$

Thus using Lemmas 11 and 13 , we see that,

$$
\left|\int_{\epsilon}^{L} t \frac{\partial^{2}}{\partial t^{2}}\left(U^{2}(y ; t) V(y ; t)\right) d t\right| \leqq A\left(f^{*}(y)\right)^{2} \phi^{*}(y),
$$

so that

$$
\begin{aligned}
\left|I_{1}\right| \leqq A \int\left(f^{*}(y)\right)^{2} \phi^{*}(y) d y & \leqq A\left(\int\left(f^{*}(y)\right)^{p} d y\right)^{2 / p}\left(\int\left(\phi^{*}(y)\right)^{q} d y\right)^{1 / q} \\
& \leqq A_{p}\|f\|_{p}^{2}
\end{aligned}
$$

by Lemma 2 . Hence,

$$
\left|I_{1}\right| \leqq A_{p}\|f\|_{p}^{2}
$$

The estimates (6.5), (6.6), and (6.7) provide an estimate for $J_{\epsilon, L},(6.4)$, and thus for $J(6.1)$.

Therefore,

$$
\|S(f)\|_{p}^{2} \leqq A_{\eta, p}\|f\|_{p}^{2}+A_{\eta, p}\|S(f)\|_{p}\|f\|_{p}
$$


and thus

$$
\|S(f)\|_{p} \leqq A_{\eta, p}^{\prime}\|f\|_{p}, \quad \text { if } p \geqq 4 .
$$

Because of (6.8) the operation is of weak type $p$, for every $p \geqq 4$. By Lemma 12 it is also of weak type 1 . Therefore using the interpolation lemma (Lemma 3), we have

$$
\|S(f)\|_{p} \leqq A_{\eta, p}\|f\|_{p}
$$

if $1<p<\infty$,

which proves the theorem.

Theorem 5. Let $f(x) \in L_{p}\left(E_{n}\right), 1<p<\infty$, and let $g(f)(x)$ be defined by (5.5). Then $\|g(f)(x)\|_{p} \leqq A_{p}\|f(x)\|_{p}, 1<p<\infty$.

Proof. The theorem follows directly from Theorem 4 and Lemma 9.

7. Converse inequalities for $g$ and $S$. The following lemma is along very classical lines.

Lemma 14. Let $U(x ; t)$ be harmonic in $x_{1}, x_{2}, \cdots, x_{n}, t, t>0$. Suppose that $U(x ; t) \rightarrow 0$ uniformly in $x$, as $t \rightarrow \infty$, and that $\int_{E_{n}}|U(x ; t)|^{p} d x \leqq A$, for $t>0,1<p<\infty$, with $A$ independent of $t$. Then $U(x ; t)$ is the Poisson integral of a function $f(x)$, and $f(x) \in L_{p}\left(E_{n}\right)$.

Proof. Since $\int_{E_{n}}|U(x ; 1 / k)|^{p} d x<\infty, k=1,2, \cdots$, then by a well-known property of weak compactness in $L_{p}, 1<p<\infty$, there exists a subsequence $\left\{1 / k_{i}\right\}$ so that $U\left(x ; 1 / k_{i}\right)$ converges weakly in $L_{p}\left(E_{n}\right)$. Let $f(x)$ denote this weak limit. Further, let $U^{\prime}(x ; t)$ be the Poisson integral of $f(x)$, and $V_{k_{i}}(x ; t)$ be the Poisson integral of $U\left(x ; 1 / k_{i}\right)$.

Then $V_{k_{i}}(x ; t)-U\left(x ;\left(1 / k_{i}\right)+t\right)$ is harmonic for $t>0$ and tends to zero as $t \rightarrow 0$, and $t \rightarrow \infty$.

Thus $U\left(x ;\left(1 / k_{i}\right)+t\right)=V_{k_{i}}(x ; t)$, and $U\left(x ; 1 / k_{i}+t\right)$ is the Poisson integral of $U\left(x ; 1 / k_{i}\right)$. However since $U\left(x ; 1 / k_{i}\right) \rightarrow f(x)$ weakly in $L_{p}$, then $U(x$; $\left.\left(1 / k_{i}\right)+t\right) \rightarrow U^{\prime}(x ; t)$ for every $x$, if $t>0$. Thus $U(x ; t)=U^{\prime}(x ; t)$, and therefore $U(x ; t)$ is the Poisson integral of $f(x)$, which proves the lemma.

The following is the basic lemma of this section:

Lemma 15. Let $U(x ; t)$ be harmonic in $x_{1} \cdots x_{n}, t, t>0$; assume $U(x ; t)$ tends to 0 uniformly in $x$ as $t \rightarrow \infty$. DeFINE:

$$
g_{r}(U)(x)=\left(\int_{0}^{\infty} t\left(\frac{\partial U(x ; t)}{\partial t}\right)^{2} d t\right)^{1 / 2} .
$$

Suppose that $g_{r}(U)(x)$ is finite for almost every $x \in E_{n}$ and is in the class $L_{p}\left(E_{n}\right)$, $1<p<\infty$. Conclusions: (1) $U(x ; t)$ is the Poisson integral of a function $f(x)$, $f(x) \in L_{p}\left(E_{n}\right)$. (2) $\|f(x)\|_{p} \leqq A_{p}\left\|g_{r}(U)\right\|_{p}$, where $A_{p}$ is independent of $U(x ; t)$.

Proof. CASE I. Assume that $U(x ; t)$ is the Poisson integral of a continuous function $f(x)$ which vanishes outside a bounded set. In this case the first 
conclusion of the lemma is certainly satisfied.

Define $h(x)$, by $h(x)=|f(x)|^{p-1} \operatorname{sign}(f(x))$. Thus $h(x)$ is also continuous, and vanishes outside a bounded set. Let $U(x ; t)$ and $V(x ; t)$ denote the Poisson integrals of $f(x)$ and $h(x)$ respectively. We note that there exists a constant $k$ so that the following are true:

$$
\begin{gathered}
|f(x)| \leqq k,|h(x)| \leqq k, \int_{E_{n}}|U(x ; t)| d x \leqq k \\
\int_{E_{n}}|V(x ; t)| d x \leqq k, \text { and, }\left|t \frac{\partial U}{\partial t}(x ; t)\right| \leqq k,\left|t \frac{\partial V}{\partial t}(x ; t)\right| \leqq k \text {, for } t>0 .
\end{gathered}
$$

The last two follow easily from Lemma 11 . We also notice both $U(x ; t)$ and $V(x ; t)$ tend to zero uniformly in $x$, as $t \rightarrow \infty$, and that $U(x ; t)$ and $V(x ; t)$ tend uniformly to $f(x)$ and $h(x)$ respectively as $t \rightarrow 0$. We add a last preliminary observation: $t(\partial U / \partial t)(x ; t)$ tends uniformly to zero as $t \rightarrow 0$. In fact, write $f(x)=f_{1}(x)+f_{2}(x)$, where $f_{1}(x)$ is sufficiently differentiable and $\left|f_{2}(x)\right|<\epsilon$.

If $U_{i}(x ; t)$ are the Poisson integrals of $f_{i}(x) i=1,2$, then $|t(\partial U / \partial t)(x ; t)|$ $\leqq\left|t\left(\partial U_{1} / \partial t\right)(x ; t)\right|+A \epsilon$, by Lemma 11 .

However, by the smoothness of $f_{1}(x)$ it is easily verified that $\mid t\left(\partial U_{1} / \partial t\right)$ $\cdot(x ; t) \mid \rightarrow 0$ uniformly in $x$, as $t \rightarrow 0$. Thus choosing $\epsilon$ appropriately we conclude that $|t(\partial U / \partial t)(x ; t)| \rightarrow 0$ uniformly as $t \rightarrow 0$. A similar conclusion, of course, holds for $|t(\partial V / \partial t)(x ; t)|$.

Now, an integration by parts establishes, if $0<\epsilon<L$,

$$
\left.\left.\int_{\epsilon}^{L} t[U(x ; t) V(x ; t)]_{t} d t=t[U(x ; t) V(x ; t)]_{t}\right]_{\epsilon}^{L}-U(x ; t) V(x ; t)\right]_{\epsilon}^{L} .
$$

By the observations which have been made in the above paragraphs, we can rewrite $(7.1)$ as

$$
\int_{\epsilon}^{L} t[U(x ; t) V(x ; t)]_{t t} d t=f(x) h(x)+J_{\epsilon}(x)+J_{L}^{\prime}(x)
$$

where $\int_{E_{n}}\left|J_{\epsilon}(x)\right| d x \rightarrow 0$ as $\epsilon \rightarrow 0$, and $\int_{E_{n}}\left|J_{L}^{\prime}(x)\right| d x \rightarrow 0$ as $L \rightarrow \infty$.

Integrate (7.2) with respect to $x$ and reverse the order of integration; then,

$$
\begin{aligned}
\int_{\epsilon}^{L} t d t \int_{E_{n}}[U(x ; t) V(x ; t)]_{t t} d x= & \int_{E_{n}} f(x) h(x) d x+\int_{E_{n}} J_{\epsilon}(x) d x \\
& +\int_{E_{n}} J_{L}^{\prime}(x) d x .
\end{aligned}
$$

However, 


$$
\begin{aligned}
& \int_{E_{n}}[U(x ; t) V(x ; t)]_{t t} d x \\
& =\int_{E_{n}}\left[U_{t t}(x ; t) V(x ; t)+2 U_{t}(x ; t) V_{t}(x ; t)+U(x ; t) V_{t t}(x ; t)\right] d x,
\end{aligned}
$$

while since $U(x ; t)$ and $V(x ; t)$ are Poisson integrals of functions in $L_{2}\left(E_{n}\right)$ it is easily verified by Plancherel's formula that

$$
\int_{E_{n}} U_{t t}(x ; t) V(x ; t) d x=\int_{E_{n}} U(x ; t) V_{t t}(x ; t) d x=\int_{E_{n}} U_{t}(x ; t) V_{t}(x ; t) d x .
$$

Thus

$4 \int_{E_{n}} \int_{\epsilon}^{L} t U_{t}(x ; t) V_{t}(x ; t) d t d x=\int_{E_{n}} f(x) h(x) d x+\int_{E_{n}} J_{\epsilon}(x) d x+\int_{E_{n}} J_{L}^{\prime}(x) d x$.

Letting $\epsilon \rightarrow 0$, and $L \rightarrow \infty$ in the above, and using Schwarz's inequality we get

$$
4 \int_{E_{n}} g_{r}(f)(x) g_{r}(h)(x) d x \geqq \int_{E_{n}} f(x) h(x) d x=\int_{E_{n}}|f(x)|^{p} d x .
$$

Here $g_{r}(f)(x)=\left(\int_{0}^{\infty} t(\partial U(x ; t) / \partial t)^{2} d t\right)^{1 / 2}$, and $g_{r}(h)(x)=\left(\int_{0}^{\infty} t(\partial V(x ; t) / \partial t)^{2} d t\right)^{1 / 2}$.

Using Hölder's inequality in (7.4) again we get, $4\left\|g_{r}(f)(x)\right\|\left\|_{p}\right\| g_{r}(h)(x) \|_{p^{\prime}}$ $\geqq \int_{E_{n}}|f|^{p} d x$ where $1 / p+1 / p^{\prime}=1$.

However, $\left\|g_{r}(h)(x)\right\|_{p^{\prime}} \leqq A_{p^{\prime}}\|h(x)\|_{p^{\prime}}$, by Theorem 4. While

$$
\left(\int_{E_{n}}|h(x)|^{p^{\prime}} d x\right)^{1 / p^{\prime}}=\left(\int|f(x)|^{(p-1) p^{\prime}} d x\right)^{1 / p^{\prime}}=\|f(x)\|_{p}^{p-1} .
$$

Thus $4\left\|g_{r}(f)(x)\right\|_{p} A_{p^{\prime}}\|f(x)\|_{p}^{p-1} \geqq\|f(x)\|_{p}^{p}$, and thus

$$
\|f(x)\|_{p} \leqq\left(1 / 4 A_{p^{\prime}}\right)\left\|g_{r}(f)(x)\right\|_{p} .
$$

Therefore the lemma is proved in this case.

CASE II. Assume that $U(x ; t)$ is the Poisson integral of a function $f(x) \in L_{p}\left(E_{n}\right)$.

Let $f_{k}(x)$ be a sequence of continuous functions vanishing outside bounded sets so that: $\left\|f(x)-f_{k}(x)\right\|_{p} \rightarrow 0$.

Then by Case I

$$
\left\|f_{k}(x)\right\|_{p} \leqq A_{p}\left\|g_{r}\left(f_{k}\right)(x)\right\|_{p}
$$

we claim that:

$$
\left|g_{r}\left(f_{k}\right)(x)-g_{r}(f)(x)\right| \leqq g_{r}\left(f_{k}-f\right)(x) .
$$

In fact, by Minkowski's inequality: $g_{r}(\alpha+\beta)(x) \leqq g_{r}(\alpha)(x)+g_{r}(\beta)(x)$, for any two functions $\alpha(x), \beta(x) \in L_{p}\left(E_{n}\right)$. Letting $\alpha=f(x)-f_{k}(x)$ and $\beta=f_{k}(x)$, we 
get $g_{r}(f)(x)-g_{r}\left(f_{k}\right)(x) \leqq g_{r}\left(f-f_{k}\right)(x)$. Letting $\alpha=f_{k}(x)-f(x), \beta=f(x)$, we get

$$
g_{r}\left(f_{k}\right)(x)-g_{r}(f)(x) \leqq g_{r}\left(f_{k}-f\right)(x)=g_{r}\left(f-f_{k}\right)(x) .
$$

Thus (7.6) is proved. By Theorem 4, however, since $\left\|f_{k}(x)-f(x)\right\|_{p} \rightarrow 0$, then $\left\|g_{r}\left(f_{k}-f\right)(x)\right\|_{p} \rightarrow 0$. Therefore combining (7.5) and (7.6) proves $\|f(x)\|_{p}$ $\leqq A_{p}\left\|g_{r}(f)(x)\right\|_{p}$, and Case II is disposed of.

CASE III. The general case. We notice that if $0<\epsilon<L<\infty$, then

$$
\begin{aligned}
|U(x ; L)-U(x ; \epsilon)| & \leqq \int_{\epsilon}^{L}\left|\frac{\partial U(x ; t)}{\partial t}\right| d t \leqq\left(\int_{\epsilon}^{L}\left(\frac{\partial U(x ; t)}{\partial t}\right)^{2} d t\right)^{1 / 2} \cdot L^{1 / 2} \\
& \leqq \epsilon^{-1 / 2} L^{1 / 2} \cdot \int_{\epsilon}^{L} t\left(\frac{\partial U(x ; t)}{\partial t}\right)^{2} d t \leqq \epsilon^{-1 / 2} L^{1 / 2} g_{r}(U)(x) \in L_{p}\left(E_{n}\right) .
\end{aligned}
$$

Thus if $\epsilon$, and $L$ are fixed, and $\bar{f}(x)=\bar{f}_{\epsilon, L}(x)=U(x, L)-U(x, \epsilon)$, then $\bar{f}(x)$ $\in L_{p}\left(E_{n}\right)$. Let $U^{\prime}(x ; t)$ be the Poisson integral of $\bar{f}(x)$. Then $U^{\prime}(x ; t)-[U(x$; $L+t)-U(x ; \epsilon+t)]$ is harmonic in $x_{1} \cdots x_{n}, t>0$, and tends to zero as $t \rightarrow 0$ and $t \rightarrow \infty$ uniformly in $x$. Hence $U^{\prime}(x ; t)-[U(x ; L+t)-U(x ; \epsilon+t)]=0$, and

$$
U^{\prime}(x ; t)=U(x ; L+t)-U(x ; \epsilon+t), \quad \text { if } t>0 .
$$

However,

$$
\begin{aligned}
& \int_{0}^{\infty} t\left(\frac{\partial U^{\prime}(x ; t)}{\partial t}\right)^{2} d t \\
& \leqq 2 \int_{0}^{\infty} t\left(\frac{\partial U(x ; L+t)}{\partial t}\right)^{2} d t+2 \int_{0}^{\infty}\left(\frac{\partial U(x ; \epsilon+t)}{\partial t}\right)^{2} d t \\
& \leqq 2 \int_{L}^{\infty}(t-L)\left(\frac{\partial U(x ; t)}{\partial t}\right)^{2} d t+2 \int_{\epsilon}^{\infty}(t-\epsilon)\left(\frac{\partial U(x ; t)}{\partial t}\right)^{2} d t \\
& \leqq 4 g_{r}^{2}(U)(x) .
\end{aligned}
$$

Thus $\left\|g_{r}\left(U^{\prime}\right)(x)\right\|_{p} \leqq 2\left\|g_{r}(U)(x)\right\|_{p}<\infty$, and we may apply Case II to the function $U^{\prime}(x ; t)$ which is the Poisson integral of $\bar{f}(x)$. Therefore

$$
\int_{E_{n}}|\bar{f}(x)|^{p} d x \leqq A_{p}^{p} \cdot 2^{p} \int_{E_{n}}\left|g_{r}(U)(x)\right|^{p} d x
$$

or

$$
\int_{E_{n}}|U(x ; \epsilon)-U(x ; L)|^{p} \leqq A_{p}^{p} \cdot 2^{p} \int_{E_{n}}\left|g_{r}(U)(x)\right|^{p} d x
$$

where $A_{p}$ is independent of $U, \epsilon$, and $L$.

Now since $U(x ; L) \rightarrow 0$ uniformly in $x$ as $L \rightarrow \infty$ then 


$$
\int_{|x| \leqq N}|U(x ; \epsilon)|^{p} d x \leqq A_{p}^{p} \cdot 2^{p} \cdot \int\left|g_{r}(U)(x)\right|^{p} d x, \quad \text { for any } N .
$$

Therefore, $\int_{E_{n}}|U(x ; \epsilon)|^{p} d x \leqq A_{p}^{p} \cdot 2^{p} \int\left|g_{r}(U)(x)\right|^{p} d x$ for any $\epsilon>0$. Hence by Lemma $14, U(x ; t)$ is the Poisson integral of a function $f(x) \in L_{p}\left(E_{n}\right)$, and $\|f(x)\|_{p}=\lim _{\epsilon \rightarrow 0}\left(\int_{E_{n}}|U(x ; \epsilon)|^{p} d x\right)^{1 / p}$.

Thus $\|f(x)\|_{p} \leqq 2 A_{p}\left\|g_{r}(U)(x)\right\|_{p}$, which concludes the proof of the lemma.

Theorem 6. Let $U(x ; t)$ be harmonic in $x_{1}, x_{2}, \cdots, x_{n}, t, t>0$ and suppose that $U(x ; t) \rightarrow 0$ uniformly in $x$ as $t \rightarrow \infty$.

Let $\left({ }^{19}\right) g(U)(x)=\left(\int_{0}^{\infty} t \operatorname{grad}^{2} U(x ; t) d t\right)^{1 / 2}$.

Suppose that $g(U)(x)$ is finite almost everywhere for $x \in E_{n}$, and that $g(U)(x)$ is in the class $L_{p}\left(E_{n}\right), 1<p<\infty$. Then we may conclude

(1) $U(x ; t)$ is the Poisson integral of a function $f(x) \in L_{p}\left(E_{n}\right)$ and (2) $\|f(x)\|_{p} \leqq A_{p}\|g(U)(x)\|_{p}$ where $A_{p}$ is independent of $U(x ; t)$.

Proof. The theorem is an immediate consequence of Lemma 15, when we notice that $g_{r}(U)(x) \leqq g(U)(x)$.

THEOREM 7. Let $U(x ; t)$ be a harmonic function in $x_{1}, x_{2}, \cdots, x_{n}, t, t>0$, and suppose that $U(x ; t) \rightarrow 0$ uniformly in $x$ as $t \rightarrow \infty$. Let

$$
S(U)(x)=\left(\iint_{W(x)}\left(\operatorname{grad}^{2} U(y ; t) / l^{n-1}\right) d y d l\right)^{1 / 2},
$$

where $W(x)$ is the "cone" $|y-x| \leqq \eta t, 0<\eta<\infty$, fixed.

Suppose $S(U)(x)$ is finite almost everywhere in $x \in E_{n}$ and $S(U)(x) \in L_{p}\left(E_{n}\right)$. Then if $1<p<\infty$,

(1) $U(x ; t)$ is the Poisson integral of a function $f(x) \in L_{p}\left(E_{n}\right)$, and

(2) $\|f(x)\|_{p} \leqq A_{p, \eta}\|S(U)(x)\|_{p}$.

Proof. The proof of this theorem follows from Theorem 6 and the fact that $g(U)(x) \leqq A_{\eta} S(U)(x)$.

This was proved in Lemma 9 when $U(x ; t)$ is the Poisson integral of a function in $L_{p}, 1 \leqq p<\infty$. The proof holds without change in the more general situation, and we shall therefore not give it.

8. Converse inequalities for $\mu$. It is our purpose to deal with an inequality of the form

$$
\|f\|_{p} \leqq A_{p}\|\mu(f)\|_{p}, \quad 1<p<\infty,
$$

where

$$
\mu(f)(x)=\left(\int_{0}^{\infty} \frac{\left|F_{t}(x)\right|^{2}}{t^{3}} d t\right)^{1 / 2} \text { and } F_{t}(x)=\int_{|y| \leqq t} f(x-y) \frac{\Omega\left(y^{\prime}\right)}{|y|^{n-1}} d y,
$$

with the kernel $\Omega$ satisfying conditions (2.5) and (2.6).

(19) As before, $\operatorname{grad}^{2} U(x ; t)=(\partial U(x ; t) / \partial t)^{2}+\sum_{i=1}^{n}\left(\partial U(x ; t) / \partial x_{i}\right)^{2}$. 
These two conditions alone are not sufficient to guarantee the validity of $(8.1)\left({ }^{20}\right)$. Instead of trying to treat the general problem of the validity of (8.1), we shall restrict our attention to a special case which may have some interest.

Define the family of kernels $\Omega_{i}(y)$ as follows

$$
\Omega_{i}(y)=\frac{y_{i}}{|y|}, \quad i=1,2, \cdots n .
$$

Define $F_{t}^{l}(x)$ by

$$
F_{t}^{i}(x)=\int_{|y| \lesssim t} \frac{\Omega_{i}(y)}{|y|^{n-1}} f(x-y) d y
$$

and

$$
\nu_{i}(f)(x)=\left(\int_{0}^{\infty} \frac{\left|F_{t}^{i}(x)\right|^{2}}{t^{3}} d t\right)^{1 / 2}
$$

also

$$
\nu(f)^{2}(x)=\sum_{i=1}^{n} \nu_{i}^{2}(f)(x)
$$

The kernels $\Omega_{i}(y)$ are odd functions, and thus satisfy the conditions of Theorem 2. Therefore,

$$
\|\nu(f)(x)\|_{p} \leqq A_{p}\|f\|_{p} \quad 1<p<\infty .
$$

We shall prove:

Theorem 8. Let $f(x) \in L_{p}\left(E_{n}\right)$, then

$$
\|f(x)\|_{p} \leqq A_{p}\|\nu(f)(x)\|_{p}, \quad 1<p<\infty .
$$

Proof. We first introduce the following weaker forms of the LittlewoodPaley $g$ function:

$$
g_{i}(f)(x)=\left(\int_{0}^{\infty} t\left(\frac{\partial U(x ; t)}{\partial x_{i}}\right)^{2} d t\right)^{1 / 2}, \quad i=1,2, \cdots n
$$

and

$$
g_{T}^{2}(f)(x)=\sum_{i=1}^{n} g_{i}^{2}(f)(x)
$$

Theorem 8 will then be a consequence of the following two lemmas $\left({ }^{21}\right)$ :

(20) It would of course be assumed that $\Omega$ is not identically zero. [11].

(21) The one-dimensional periodic analogues of Lemmas 16 and 17 are due to A. Zygmund 
Lemma 16. $g_{i}(f)(x) \leqq A \nu_{i}(f)(x), i=1,2, \cdots, n$ where $A$ is a constant independent of $f(x)$.

Lemma 17. Let $f(x) \in L_{p}\left(E_{n}\right)$, then

$$
\|f(x)\|_{p} \leqq A_{p}\left\|g_{T}(f)(x)\right\|_{p}, \quad 1<p<\infty .
$$

Proof of Lemma 16. Using formula (5.1) we see that

$$
-\frac{1}{(n+1)} \cdot \frac{1}{c_{n}} \frac{\partial U(x ; t)}{\partial x_{i}}=t \int_{E_{n}} \frac{|y| \Omega_{i}(y) f(x-y)}{\left(|y|^{2}+t^{2}\right)^{(n+3) / 2}} d y
$$

where $\Omega_{i}(y)=y_{i} /|y|$. Let $|y|=r$, then

$t \int_{E_{n}} \frac{|y| \Omega_{i}(y) f(x-y)}{\left(|y|^{2}+t^{2}\right)^{(n+3) / 2}} d y=t \int_{0}^{\infty}\left(\int_{\Sigma} f\left(x-r y^{\prime}\right) \Omega_{i}\left(y^{\prime}\right) d \Sigma\right) \frac{r^{n} d r}{\left(r^{2}+t^{2}\right)^{(n+3) / 2}}$.

Here, $\Sigma$ is the unit sphere in the $n$-dimensional space of $y$. Now by integrating by parts with respect to $r$ we obtain

$$
\begin{aligned}
& \left(\int_{\epsilon}^{1 / \epsilon}\left(\int_{\Sigma} f\left(x-r y^{\prime}\right) \Omega\left(y^{\prime}\right) d \Sigma\right) \frac{r^{n} d r}{\left(r^{2}+t^{2}\right)^{(n+3) / 2}}\right. \\
& =-\int_{\epsilon}^{1 / \epsilon} F_{s}^{i}(x) K(s, t) d s+F_{1 / \epsilon}^{i}(x) \frac{\epsilon^{-n}}{\left(\epsilon^{-2}+t^{2}\right)^{(n+3) / 2}}-F_{\epsilon}^{i}(x) \frac{\epsilon^{n}}{\left(\epsilon^{2}+t^{2}\right)^{(n+3) / 2}}
\end{aligned}
$$

where $F_{s}^{\imath}(x)=\int_{|y| \leqq s} f(x-y) \Omega_{i}(y) /|y|^{n-1} d y$, and

$$
K(s, t)=\frac{n s^{n-1}}{\left(s^{2}+t^{2}\right)^{(n+3) / 2}}-(n+3) \frac{s^{n+1}}{\left(s^{2}+t^{2}\right)^{(n+5) / 2}} .
$$

Now if $t>0$, the last two terms in (8.5) tend to zero for almost every $x$, as $\epsilon \rightarrow 0\left({ }^{22}\right)$. Thus (8.5) becomes

$$
-\frac{1}{(n+1)} \cdot \frac{1}{c_{n}} \cdot \frac{\partial U(x ; t)}{\partial x_{i}}=t \int_{0}^{\infty} F_{s}^{i}(x) K(s, t) d s .
$$

Now for $K(s, t)$ we make the following obvious estimates:

$$
\begin{array}{ll}
|K(s, t)| \leqq A / t^{4}, & \text { if } s \leqq t \\
|K(s, t)| \leqq A / s^{4}, & \text { if } s \geqq t .
\end{array}
$$

Thus,

$$
\left|\frac{\partial U(x ; t)}{\partial x_{i}}\right| \leqq A t^{-3} \int_{0}^{t}\left|F_{s}^{i}(x)\right| d s+A t \int_{t}^{\infty} \frac{\left|F_{s}^{i}(x)\right| d s}{s^{4}} .
$$

Using Schwarz's inequality, we obtain

(22) In fact, by Lemma $6\left|\epsilon^{-1} F_{\epsilon}(x)\right|$ is bounded in $\epsilon$, for almost every $x$. 
$t \cdot\left(\frac{\partial U(x ; t)}{\partial x_{i}}\right)^{2}$

$$
\begin{aligned}
& \leqq 2 A^{2} t^{-6} \cdot t \int_{0}^{t}\left|F_{s}^{i}(x)\right|^{2} d s \int_{0}^{t} d s+2 A^{2} t^{2} \cdot t \int_{t}^{\infty} \frac{\left|F_{s}^{i}(x)\right|^{2}}{s^{4}} d s\left(\int_{t}^{\infty} \frac{d s}{s^{4}}\right) \\
& \leqq A^{\prime}\left(t^{-4} \int_{0}^{t}\left|F_{s}^{i}(x)\right|^{2} d s+\int_{t}^{\infty} \frac{\left|F_{s}^{i}(x)\right|^{2}}{s^{4}} d s\right) .
\end{aligned}
$$

Integrating with respect to $t$, and inverting the order of integration we obtain:

$$
\begin{gathered}
\int_{0}^{\infty} t\left(\frac{\partial U(x ; t)}{\partial x_{i}}\right)^{2} d t \leqq A^{\prime} \int_{0}^{\infty} \frac{d t}{t^{4}} \int_{0}^{t}\left|F_{s}^{2}(x)\right|^{2} d s+A^{\prime} \int_{0}^{\infty} d t \int_{t}^{\infty} \frac{\left|F_{s}^{i}(x)\right|^{2}}{s^{4}} d s \\
\leqq A^{\prime} \int_{s}^{\infty}\left|F_{s}^{i}(x)\right|^{2}\left(\int_{s}^{\infty} \frac{d t}{t^{4}}\right) d s+A^{\prime} \cdot \int_{0}^{\infty} \frac{\left|F_{s}^{i}(x)\right|^{2}}{s^{4}}\left(\int_{0}^{s} d t\right) d s \\
\leqq A \int_{0}^{\infty} \frac{\left|F_{s}^{i}(x)\right|^{2}}{s^{3}} d s .
\end{gathered}
$$

Thus $g_{i}^{2}(f)(x) \leqq A \nu_{\imath}^{2}(f)(x)$, and Lemma 16 is proved.

Proof of Lemma 17. Let $R_{i}(\cdot)$ denote the "Riesz-transforms" defined by (5.2), and let $f_{i}(x)=R_{i}(f)(x)$. Then the following identity holds

$$
\sum_{i=1}^{n} R_{i}\left(f_{i}\right)(x)=-f(x) .
$$

The above is proved in [4] when $f(x) \in L_{2}\left(E_{n}\right)$ by means of the Fourier transform. (8.7) may then be established for any $f \in L_{p}\left(E_{n}\right)$ by using the boundedness of the transformations $R_{i}(f)$, for $f \in L_{p}\left(E_{n}\right)$, (Lemma 4). Because of (5.3)

$$
g_{r}\left(f_{i}\right)(x) \leqq g_{T}(f)(x),
$$

where $g_{r}(f)(x)=\left(\int_{0}^{\infty} t(\partial U(x ; t) / \partial t)^{2} d t\right)^{1 l 2}$, and hence by Lemma 15 ,

$$
\left\|f_{i}(x)\right\|_{p} \leqq A_{p}\left\|g_{r}\left(f_{i}\right)(x)\right\|_{p} \leqq A_{p}\left\|g_{T}(f)(x)\right\|_{p}, \quad 1<p<\infty .
$$

However, (8.7) and Lemma 4 imply that:

$$
\|f(x)\|_{p} \leqq A_{p} \sum_{i=1}^{n}\left\|f_{i}(x)\right\|_{p}, \quad 1<p<\infty .
$$

Thus combining (8.8), and (8.9) we obtain

$$
\|f(x)\|_{p} \leqq A_{p}\left\|g_{T}(f)(x)\right\|_{p}, \quad 1<p<\infty .
$$




\section{This proves Lemma 17, and thus Theorem 8 .}

\section{REFERENCES}

1. A. P. Calderón, On the theorem of Marcinkiewicz and Zygmund, Trans. Amer. Math. Soc. vol. 68 (1950) pp. 55-61.

2. A. P. Calderón and A. Zygmund, On the existence of certain singular integrals, Acta Math. vol. 88 (1952) pp. 85-139.

3. — On singular integrals, Amer. J. Math. vol. 78 (1956) pp. 289-309.

4. J. Horvàth, Sur les fonctions conjuguées a plusieurs variables, Nederl. Akad. Wetensch. vol. 15 (1953) pp. 17-29.

5. J. E. Littlewood and R. E. A. C. Paley, Theorems on Fourier series and power series, Part I, J. London Math. Soc. vol. 6 (1931) pp. 230-233; Part II, Proc. London Math. Soc. vol. 42 (1937) pp. 52-89; Part III, ibid, vol. 43 (1937) pp. 105-126.

6. N. Lusin, Sur une propriété des fonctions a carré sommable, Bull. Calcutta Math. Soc. vol. 20 (1930) pp. 139-154.

7. J. Marcinkiewicz, Sur quelques integrales de type de Dini, Annales de la Société Polonaise, vol. 17 (1938) pp. 42-50.

8. - Sur les series de Fourier, Fund. Math. vol. 27 (1936) pp. 38-69.

9. J. Marcinkiewicz and A. Zygmund, On a theorem of Lusin, Duke Math. J. vol. 4 (1938) pp. $473-485$.

10. D. Waterman, On an integral of Marcinkiewicz, Proceedings of the International Congress of Mathematicians, vol. 2, 1954, pp. 185-186; also On functions analytic in a halfplane, Trans. Amer. Math. Soc. vol. 81 (1956) pp. 167-194. 204.

11. A. Zygmund, On certain integrals, Trans. Amer. Math. Soc. vol. 55 (1944) pp. 170-

12. - Proof of a theorem of Littlewood and Paley, Bull. Amer. Math. Soc. vol. 51 (1945) pp. 439-446.

13. - On a theorem of Marcinkiewicz concerning interpolation of operations, J. Math. Pures Appl. vol. 35 (1956) pp. 223-248.

14. - Trigonometrical series, 2d. ed., to appear.

Massachusetts Institute of Technology,

Cambridge, Mass. 